\title{
Reductive Cross-Aldol Reaction Using Bromoaldehyde and an Aldehyde Mediated by Germanium(II): One-Pot, Large-Scale Protocol
}

Makoto Yasuda, Shin-ya Tanaka, and Akio Baba*

Department of Molecular Chemistry and Handai Frontier Research Center, Graduate School of Engineering, Osaka University, 2-1 Yamadaoka, Suita, Osaka 565-0871, Japan

\section{Supporting Information}

General. IR spectra were recorded as thin films or as solids in $\mathrm{KBr}$ pellets on a HORIBA FT720 spectrophotometer. ${ }^{1} \mathrm{H}$ and ${ }^{13} \mathrm{C}$ NMR spectra were obtained with TMS as internal standard. Techniques of HMBC or HMQC were employed to assign signals. Mass spectra were recorded on a JEOL JMS-DS303 spectrometer. Column chromatography was performed on silica gel. Bulb-to-bulb distillation (Kugelrohr) was accomplished in a Sibata GTO-250RS at the oven temperature and pressure indicated. Yields were determined by ${ }^{1} \mathrm{H}$ NMR using internal standards.

Materials. THF, $\mathrm{Et}_{2} \mathrm{O}$, hexane were distilled from sodium with benzophenone. DMF was predried by MS3A and distilled. Dichloromethane, dehydrated purchased from Wako Pure Chemical Ind. Ltd. (stabilized with 2-methyl-2-butene) was used without purification. Methanol was dried over MS3A. $\mathrm{Zn}, \mathrm{SmI}_{2}, \mathrm{CrCl}_{2}$, and $\mathrm{SnCl}_{2}$ were commercially available. $\mathrm{GeCl}_{2}$-dioxane was prepared by known method. ${ }^{1}$ The aldehydes 2a-e and heptanal $\mathbf{7}$ were commercially available. The bromoaldehydes $\mathbf{1 a}$ and $\mathbf{1 b}$ were prepared by known procedure., ${ }^{2,3}$ The bromoaldehyde 1c was prepared according to the known procedure. ${ }^{4}$ To a stirred solution of 3phenylpropanal $(120 \mathrm{mmol})$ and DMSO $(125 \mathrm{mmol})$ in dry acetonitrile $(100 \mathrm{~mL})$ was slowly added $\mathrm{Me}_{3} \mathrm{SiBr}(130 \mathrm{mmol})$ for $15 \mathrm{mim}$. The reaction temperature was kept at $20^{\circ} \mathrm{C}$ by external cooling for $45 \mathrm{~min}$. Volatiles were directly removed under reduced pressure at $\mathrm{rt}$. The distillation $\left(73-76{ }^{\circ} \mathrm{C} / 0.2 \mathrm{mmHg}\right)$ gave the product $1 \mathrm{c}(22.8 \mathrm{~g}, 89 \%) .{ }^{5}$ The brominated bis aldehyde 5 was prepared as shown below.

\section{4-(2-Formylphenyl)butanal}

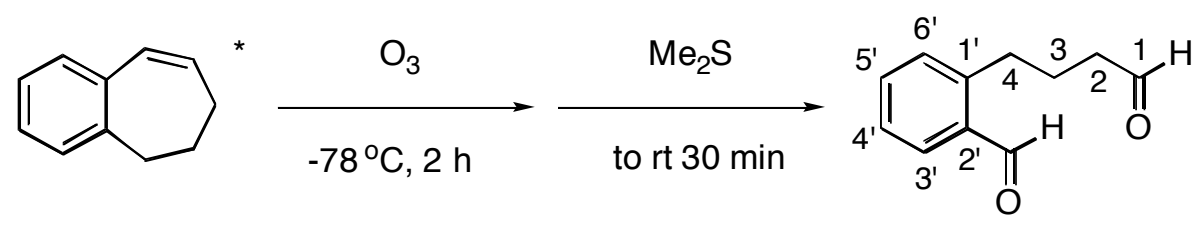

A solution of the 6,7-dihydro-5H-benzocycloheptene (benzosuberene) ${ }^{6}(5.7 \mathrm{~g}, 39.5 \mathrm{mmol})$ in $\mathrm{CH}_{2} \mathrm{Cl}_{2}(140 \mathrm{~mL})$ and $\mathrm{MeOH}(17 \mathrm{~mL})$ was cooled to $-78{ }^{\circ} \mathrm{C}$, and $\mathrm{O}_{3}$ was bubbled until the reaction mixture was light blue (excess $\mathrm{O}_{3}$ ). The resulting mixture was stirred for $15 \mathrm{~min}$ at room 
temperature after $\mathrm{Me}_{2} \mathrm{~S}(5 \mathrm{~mL}, 68 \mathrm{mmol})$ was added. Then the solution was washed with aqueous $\mathrm{NaHCO}_{3}$ (saturated; $100 \mathrm{~mL}, 20 \mathrm{~mL}$ x 2), and aqueous $\mathrm{FeSO}_{4}-7 \mathrm{H}_{2} \mathrm{O}(0.04 \mathrm{M} ; 100 \mathrm{~mL}$ ), and the organic layer was dried over $\mathrm{MgSO}_{4}$. The solvent was evaporated under reduced pressure and the residue was purified by distillation to give a pure product, containing a trace amount of acid (4.2 g, 23.7 mmol, 60\%): bp: $113{ }^{\circ} \mathrm{C} / 0.8 \mathrm{mmHg}$; IR: (neat) 1697 (2'-CHO), $1720\left(\mathrm{C}_{1} \mathrm{HO}\right) \mathrm{cm}^{1} ;{ }^{1} \mathrm{H}$ NMR: (400 MHz, $\mathrm{CDCl}_{3}$ ) 10.22 (s, 1H, 2'-CHO), 9.79 (t, J=1.5 Hz, 1H, 1-H), 7.83-7.81 (m, 1H, 3'-H), 7.54-7.50 (m, 1H, 5'-H), 7.43-7.39 (m, 1H, 4'-H), 7.29-7.27 (m, 1H, 6'-H), 3.07 (t, $J$ $\left.=7.8 \mathrm{~Hz}, 2 \mathrm{H}, 4-\mathrm{H}_{2}\right), 2.53\left(\mathrm{td}, J=7.3,1.5 \mathrm{~Hz}, 2 \mathrm{H}, 2-\mathrm{H}_{2}\right), 1.95\left(\mathrm{tt}, J=7.8,7.3 \mathrm{~Hz}, 2 \mathrm{H}, 3-\mathrm{H}_{2}\right) ;{ }^{13} \mathrm{C}$ NMR: (67.9 MHz, CDCl M $_{3} 201.97$ (d,C-1), 192.54 (d, 2'-CHO), 143.82 (s, C-1'), 133.68 (d, C5'), 133.58 (d, $\left.{ }^{2} J_{\mathrm{CH}}, \mathrm{C}^{\prime} 2^{\prime}\right), 133.09$ (t, C-3'), 131.01 (d, C-6'), 126.72 (d, C-4'), 43.23 (td, C-2), 31.87 (t, C-4), 24.01 (t, C-3); MS: (EI, $70 \mathrm{eV}) \mathrm{m} / \mathrm{z} 176\left(\mathrm{M}^{+}, 10\right), 158\left(\mathrm{M}^{+}-\mathrm{H}_{2} \mathrm{O}, 38\right), 133\left(\mathrm{M}^{+}\right.$$\left.\mathrm{CH}_{2} \mathrm{CH}_{2} \mathrm{CHO}, 100\right), 129\left(\mathrm{M}^{+}-\mathrm{H}_{2} \mathrm{O}-\mathrm{CHO}, 78\right), 104$ (Ph - CO, 60), $91\left(\mathrm{PhCH}_{2}, 55\right)$; HRMS: (EI, $70 \mathrm{eV}$ ) calcd for $\mathrm{C}_{11} \mathrm{H}_{12} \mathrm{O}_{2} 176.0837\left(\mathrm{M}^{+}\right)$found for $\mathrm{m} / \mathrm{z}$ 176.0833. Anal. Calcd for $\mathrm{C}_{11} \mathrm{H}_{12} \mathrm{O}_{2}: \mathrm{C}$, 74.98; H, 6.86. Found C, 75.08; H, 6.81.

\section{2-Bromo-4-(2-formylphenyl)butanal (5)}<smiles>O=CC(Br)CCc1ccccc1</smiles>

To the solution of 4-(2-formylphenyl)butanal $(6.0 \mathrm{mmol})$ and DMSO $(6.7 \mathrm{mmol})$ in $\mathrm{MeCN}$ $(12 \mathrm{~mL})$ was slowly added $\mathrm{Me}_{3} \mathrm{SiBr}(6.7 \mathrm{mmol})$. The reaction was slightly exothermic and was kept room temperature by water bath. The resulting mixture was stirred for $70 \mathrm{~min}$, and then quenched by water $(50 \mathrm{~mL})$, extracted with $\mathrm{Et}_{2} \mathrm{O}$, and the organic layer was dried over $\mathrm{MgSO}_{4}$. The solvent was evapolated under reduced pressure, and the crude product was quickly used in the subsequent reaction: IR: (neat) $1697\left(2^{\prime}-\mathrm{CHO}\right), 1724\left(\mathrm{C}_{1} \mathrm{HO}\right) \mathrm{cm}^{-1}$ : ${ }^{1} \mathrm{H} \mathrm{NMR}$ : $(600 \mathrm{MHz}$, $\left.\mathrm{CDCl}_{3}\right) 10.18$ (s, 1H, 2'-CHO), 9.48 (d, $\left.J=2.4 \mathrm{~Hz}, 1 \mathrm{H}, 1-\mathrm{H}\right), 7.84-7.81$ (m, 1H, 3'-H), 7.56-7.52 (m, 1H, 5'-H), 7.47-7.44 (m, 1H, 4'-H), 7.34-7.32 (m, 1H, 6'-H), 4.27 (ddd, $J=8.3,5.9,2.4 \mathrm{~Hz}$, $1 \mathrm{H}, 2-\mathrm{H}), 3.30-3.24\left(\mathrm{ddd}, J=13.2,9.5,5.6 \mathrm{~Hz}, 1 \mathrm{H}, 4-\mathrm{H}^{\mathrm{A}}\right), 3.19-3.12(\mathrm{ddd}, J=13.2,9.5,6.3 \mathrm{~Hz}$, $\left.1 \mathrm{H}, 4-\mathrm{H}^{\mathrm{B}}\right), 2.42-2.34\left(\mathrm{dddd}, J=14.7,9.5,6.3,5.9 \mathrm{~Hz}, 1 \mathrm{H}, 3-\mathrm{H}^{\mathrm{A}}\right), 2.24-2.17(\mathrm{dddd}, J=14.7,9.5$, 8.3, 5.6 Hz, 1H, 3-H $\left.{ }^{\mathrm{B}}\right) ;{ }^{13} \mathrm{C}$ NMR: (150 MHz, $\left.\mathrm{CDCl}_{3}\right) 192.88$ (-, 2'-CHO), 192.32 (-, C-1), 142.18 (+, C-1'), 134.42 (-, C-3'), 133.91 (-, C-4'), 133.80 (+, C-2'), 131.38 (-, C-6'), 127.30 (-, C-5'), 54.61 (-, C-2), 33.03 (+, C-3), 30.39 (+, C-4); MS: (CI, $70 \mathrm{eV}) \mathrm{m} / \mathrm{z} 257\left(\mathrm{M}^{+}+3,74\right), 255$ $\left(\mathrm{M}^{+}+1,81\right), 239\left(\mathrm{M}^{+}+3-\mathrm{H}_{2} \mathrm{O}, 20\right), 237\left(\mathrm{M}^{+}+1-\mathrm{H}_{2} \mathrm{O}, 18\right), 221\left(\mathrm{M}^{+}+3-2 \mathrm{H}_{2} \mathrm{O}, 18\right), 219\left(\mathrm{M}^{+}+\right.$ $\left.1-2 \mathrm{H}_{2} \mathrm{O}, 29\right), 207\left(\mathrm{M}^{+}-\mathrm{CHO}-\mathrm{H}_{2} \mathrm{O}, 87\right), 175\left(\mathrm{M}^{+}-\mathrm{HBr}+1,68\right), 159(100), 147\left(\mathrm{M}^{+}-\mathrm{CHO}-\mathrm{Br}\right.$ $+1,25), 129\left(\mathrm{M}^{+}-\mathrm{CHO}-\mathrm{Br}-\mathrm{H}_{2} \mathrm{O}+1,52\right)$; HRMS: $(\mathrm{CI}, 70 \mathrm{eV})$ calcd for $\mathrm{C}_{11} \mathrm{H}_{12}{ }^{81} \mathrm{BrO}_{2}$ $257.0021\left(\mathrm{M}^{+}+3\right)$ found for $\mathrm{m} / \mathrm{z} 256.9991$, calcd for $\mathrm{C}_{11} \mathrm{H}_{12}{ }^{79} \mathrm{BrO}_{2} 255.0021\left(\mathrm{M}^{+}+1\right)$ found for $\mathrm{m} / \mathrm{z} 254.9950$.

Reaction of Bromoheptanal (1a) with Benzaldehyde (2a) in the presence of Zn (Table 1, entry 1). To a stirred solution of 2-bromoheptanal $1 \mathbf{a}(1 \mathrm{mmol})$ and benzaldehyde $\mathbf{2 a}(1 \mathrm{mmol})$ in dry THF $(2 \mathrm{~mL})$ was added $\mathrm{Zn}$ power $(1 \mathrm{mmol})$ at room temperature. The reaction was stirred 
at $68{ }^{\circ} \mathrm{C}$ for $2 \mathrm{~h} . \mathrm{H}_{2} \mathrm{O}(10 \mathrm{~mL})$ was added to the reaction mixture. The resulting mixture was extracted with $\mathrm{Et}_{2} \mathrm{O}$, and organic layer was dried over $\mathrm{MgSO}_{4}$. The complicated mixture was observed by ${ }^{1} \mathrm{H}$ NMR with recovering of benzaldehyde $\mathbf{2 a}(47 \%)$. The bromoaldehyde 1a was totally consumed.

Reaction of Bromoheptanal (1a) with Benzaldehyde (2a) in the presence of $\mathrm{SmI}_{2}$ (Table 1, entry 2). To a stirred solution of $\operatorname{SmI}_{2}(0.1 \mathrm{M}$ in THF, $20 \mathrm{~mL})$ was slowly added mixture of 2bromoheptanal 1a $(1 \mathrm{mmol})$ and benzaldehyde $\mathbf{2 a}(1 \mathrm{mmol})$ in dry $\mathrm{THF}(2 \mathrm{~mL})$ at $-78{ }^{\circ} \mathrm{C}$ for 10 min. The reaction was stirred at $-78^{\circ} \mathrm{C}$ for $2 \mathrm{~h}$. Aqueous $\mathrm{NaHCO}_{3}$ (saturated; $20 \mathrm{~mL}$ ) was added to the reaction mixture. The resulting mixture was extracted with $\mathrm{Et}_{2} \mathrm{O}$, and organic layer was dried over $\mathrm{MgSO}_{4}$. The complicated mixture was observed by ${ }^{1} \mathrm{H}$ NMR with recovering of benzaldehyde $\mathbf{2 a}(25 \%)$. The bromoaldehyde 1a was totally consumed.

Reaction of Bromoheptanal (1a) with Benzaldehyde (2a) in the presence of $\mathrm{CrCl}_{2}$ (Table $\mathbf{1}$, entry 3). To a stirred suspension of $\mathrm{CrCl}_{2}(1.46 \mathrm{mmol})$ and benzaldehyde $2 \mathbf{a}(0.5 \mathrm{mmol})$ in dry THF (2 mL) was added 2-bromoheptanal $1 \mathbf{a}(0.47 \mathrm{mmol})$ at room temperature. The reaction was stirred at room temperature for $14 \mathrm{~h} . \mathrm{H}_{2} \mathrm{O}(10 \mathrm{~mL})$ was added to the reaction mixture. The resulting mixture was extracted with $\mathrm{Et}_{2} \mathrm{O}$, and organic layer was dried over $\mathrm{MgSO}_{4}$. The complicated mixture was observed by ${ }^{1} \mathrm{H}$ NMR with recovering of benzaldehyde $\mathbf{2 a}(14 \%)$. The bromoaldehyde 1a was totally consumed.

Reaction of Bromoheptanal (1a) with Benzaldehyde (2a) in the presence of $\mathrm{SnCl}_{2}\left(\mathrm{Table}^{1}\right.$, entry 4). To a stirred solution of $\mathrm{SnCl}_{2}(0.68 \mathrm{mmol})$ and benzaldehyde $\mathbf{2 a}(0.67 \mathrm{mmol})$ in dry THF (2 mL) was added 2-bromoheptanal $1 \mathrm{a}(0.68 \mathrm{mmol})$ at room temperature. The reaction was stirred at room temperature for $2 \mathrm{~h}$. Aqueous $\mathrm{NH}_{4} \mathrm{~F}(15 \%, 10 \mathrm{~mL})$ was added to the reaction mixture. The resulting mixture was extracted with $\mathrm{Et}_{2} \mathrm{O}$, and organic layer was dried over $\mathrm{MgSO}_{4}$. The recovery of bromoaldehyde 1a (65\%) and benzaldehyde $\mathbf{2 a}(49 \%)$ were found by ${ }^{1} \mathrm{H}$ NMR without any other products.

Reaction of Bromoheptanal (1a) with Benzaldehyde (2a) in the presence of $\mathbf{G e C l}_{2}$-dioxane (Table 1, entry 5). To a stirred solution of $\mathrm{GeCl}_{2}$-dioxane $(0.6 \mathrm{mmol})$ and benzaldehyde $\mathbf{2 a}(0.6$ $\mathrm{mmol})$ in dry THF $(2 \mathrm{~mL})$ was added 2-bromoheptanal $1 \mathbf{a}(0.6 \mathrm{mmol})$ at room temperature. The reaction was stirred at room temperature for $2 \mathrm{~h} . \mathrm{H}_{2} \mathrm{O}(10 \mathrm{~mL})$ was added to the reaction mixture. The resulting mixture was extracted with $\mathrm{Et}_{2} \mathrm{O}$, and organic layer was dried over $\mathrm{MgSO}_{4}$. The aldol product 3 was observed by ${ }^{1} \mathrm{H}$ NMR in $60 \%$ yield (syn:anti $\left.=64: 36\right)^{7}$ with recovering of benzaldehyde 2a (23\%). A small amount of heptanal which derived from reduction of $\mathbf{1 a}$ was observed (14\%).

General Procedure for Reductive Aldol Reaction Using $\mathrm{GeCl}_{2}$-dioxane with $\mathrm{MeOH}$ quenching (Tables 2 and 3). To a stirred suspension of $\mathrm{Bu}_{4} \mathrm{NBr}(0.006 \mathrm{mmol}), \mathrm{GeCl}_{2}$-dioxane complex $(0.6 \mathrm{mmol})$ and aldehyde $2(0.6 \mathrm{mmol})$ in solvent $(2 \mathrm{~mL})$ was added bromoaldehyde 1 $(0.6 \mathrm{mmol})$ under nitrogen. The reaction mixture was stirred under the reaction conditions noted in the text. Methanol $(8 \mathrm{~mL})$ was added and the resulting solution was stirred for additional $1 \mathrm{~h}$ at 
room temperature. Then aqueous $\mathrm{NaHCO}_{3}$ (saturated; $5 \mathrm{~mL}$ ) was added to the reaction mixture. The resulting mixture was extracted with $\mathrm{Et}_{2} \mathrm{O} /$ hexane $(4 / 1)$, and organic layer was dried over $\mathrm{MgSO}_{4}$. The procedures of further purification for new compounds are shown in the Product Data section.

Product Data. The spectral data of 4 aa was in an excellent agreement with the reported data. ${ }^{7}$ Spectral data for the products, 4ab, 4ac, 4ba, 4ca, 4cd, 4ce, 4cb, 4cc, and 6 are shown below. The experiments for the isolation of the products were employed in about fivefold scale.

Determination of Stereochiemistry of the Products. The relative stereoconfiguration of the products 4 was determined based on the coupling constant between hydrogens on chiral centers. The products exist as intramolecularly hydrogen bonded structures with the sixmembered ring in chair conformation. ${ }^{8}$ The substituents on the ring tend to locate in equatorial position except one of the two $\mathrm{MeO}$ groups of acetal moiety because of anomeric effect. ${ }^{9}$ The relative stereoconfiguration of $\mathbf{6}$ was determined by X-ray structural analysis.

$\left(6 R^{*}, 7 S^{*}\right)$-7-Dimethoxymethyl-6-dodecanol (syn-4ab).
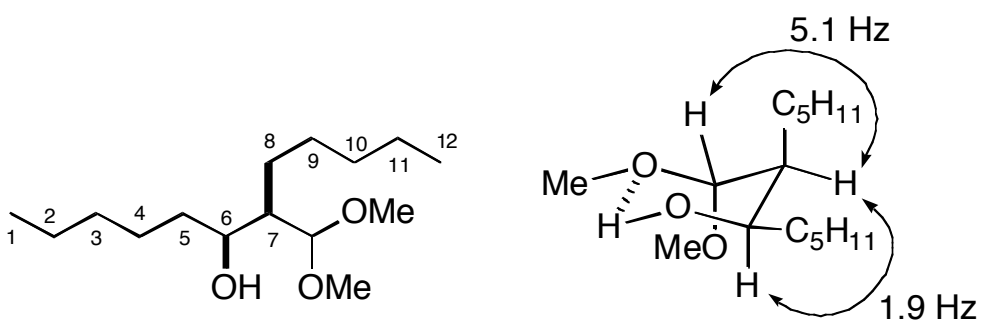

According to the general procedure, this compound was prepared from $\mathbf{1 a}, \mathbf{2 b}, \mathrm{GeCl}_{2}$-dioxane, and $\mathrm{Bu}_{4} \mathrm{NBr}$ in $\mathrm{Et}_{2} \mathrm{O}$ to give the product as a colorless liquid after column chromatography (hexane/ethyl acetate, 95/5) on silica gel (BW-300): IR: (neat) $3521(\mathrm{OH}) \mathrm{cm}^{-1}$; ${ }^{1} \mathrm{H}$ NMR: (270 $\mathrm{MHz}, \mathrm{CDCl}_{3}$ ) syn (major) $4.33\left(\mathrm{~d}, J=5.1 \mathrm{~Hz}, 1 \mathrm{H}, 7-\mathrm{CH}(\mathrm{OMe})_{2}\right), 3.89-3.79(m, 1 \mathrm{H}, 6-\mathrm{H}), 3.44$ $\left(\mathrm{s}, 3 \mathrm{H}, \mathrm{OMe}^{\mathrm{A}}\right), 3.37\left(\mathrm{~s}, 3 \mathrm{H}, \mathrm{OMe}^{\mathrm{B}}\right), 2.97\left(\mathrm{~d}, J=4.9 \mathrm{~Hz}, 1 \mathrm{H}, \mathrm{OH}, \mathrm{D}_{2} \mathrm{O}\right.$-exchangeable $), 1.75-1.65$ (m, 1H, 7-H), 1.58-1.17 (m, 16H, 2- $\mathrm{H}_{2}, 3-\mathrm{H}_{2}, 4-\mathrm{H}_{2}, 5-\mathrm{H}_{2}, 8-\mathrm{H}_{2}, 9-\mathrm{H}_{2}, 10-\mathrm{H}_{2}$, and 11- $\mathrm{H}_{2}$ ), 0.97$0.81\left(\mathrm{~m}, 6 \mathrm{H}, 1-\mathrm{H}_{3}\right.$, and 12- $\left.\mathrm{H}_{3}\right)$; ${ }^{1} \mathrm{H} \mathrm{NMR}:\left(600 \mathrm{MHz}, \mathrm{CDCl}_{3}\right.$, small amount of $\left.\mathrm{D}_{2} \mathrm{O}\right) 4.33(\mathrm{~d}, J=$ $\left.5.1 \mathrm{~Hz}, 1 \mathrm{H}, 7-\mathrm{CH}(\mathrm{OMe})_{2}\right), 3.83$ (ddd, $\left.J=9.0,4.2,1.9 \mathrm{~Hz}, 1 \mathrm{H}, 6-\mathrm{H}\right), 3.44\left(\mathrm{~s}, 3 \mathrm{H}, \mathrm{OMe}^{\mathrm{A}}\right), 3.37$ (s, $3 \mathrm{H}, \mathrm{OMe}^{\mathrm{B}}$ ), $1.70(\mathrm{tdd}, J=7.3,5.1,1.9 \mathrm{~Hz}, 1 \mathrm{H}, 7-\mathrm{H}), 1.54-1.23\left(\mathrm{~m}, 16 \mathrm{H}, 2-\mathrm{H}_{2}, 3-\mathrm{H}_{2}, 4-\mathrm{H}_{2}, 5-\mathrm{H}_{2}\right.$, 8- $\mathrm{H}_{2}, 9-\mathrm{H}_{2}, 10-\mathrm{H}_{2}$, and 11- $\left.\mathrm{H}_{2}\right), 0.95-0.87\left(\mathrm{~m}, 6 \mathrm{H}, 1-\mathrm{H}_{3}\right.$, and $\left.12-\mathrm{H}_{3}\right) ;{ }^{13} \mathrm{C} \mathrm{NMR}:(67.9 \mathrm{MHz}$, $\left.\mathrm{CDCl}_{3}\right) 108.10\left(\mathrm{~d}, 13-\mathrm{CH}(\mathrm{OMe})_{2}\right), 70.60(\mathrm{~d}, \mathrm{C}-6), 55.90\left(\mathrm{q}, \mathrm{OMe}^{\mathrm{A}}\right), 53.78\left(\mathrm{q}, \mathrm{OMe}^{\mathrm{B}}\right), 45.06(\mathrm{~d}$, C-7), 33.51 (t, C-5), 32.08 (t, C-10), 31.80 (t, C-3), 27.96 (t, C-9), 26.17 (t, C-4), 24.58 (t, C-8), 22.57 (t, C-2), 22.44 (t, C-11), 13.95 (q), 13.93 (q); MS: (CI, 70 eV) m/z $243\left(\mathrm{M}^{+}-\mathrm{H}_{2} \mathrm{O}+1,4\right)$, $229\left(\mathrm{M}^{+}\right.$- OMe, 9), $211\left(\mathrm{M}^{+}-\mathrm{H}_{2} \mathrm{O}-\mathrm{OMe}, 12\right), 197\left(\mathrm{M}^{+}-(\mathrm{OMe})_{2}+1,13\right), 129\left(\mathrm{M}^{+}-\right.$

$\left.\mathrm{C}_{5} \mathrm{H}_{11} \mathrm{CHOH}-\mathrm{OMe}+1,100\right), 115\left(\mathrm{M}^{+}-\mathrm{C}_{5} \mathrm{H}_{11}-\mathrm{CH}(\mathrm{OMe})_{2}+1,28\right), 89\left(\mathrm{CHCH}(\mathrm{OMe})_{2}+1\right.$, 14); HRMS: (CI, $70 \mathrm{eV})$ calcd for $\mathrm{C}_{15} \mathrm{H}_{31} \mathrm{O}_{2} 243.2264\left(\mathrm{M}^{+}-\mathrm{H}_{2} \mathrm{O}+\mathrm{H}\right)$ found for $\mathrm{m} / \mathrm{z} 243.2326$. Anal. Calcd for $\mathrm{C}_{15} \mathrm{H}_{32} \mathrm{O}_{3}$ (260.41): C, 69.18; H, 12.39. Found C, 69.14; H, 12.12. 
$\left(6 S^{*}, 7 S^{*}\right)-7-D i m e t h o x y m e t h y l-6-d o d e c a n o l ~(a n t i-4 a b)$.
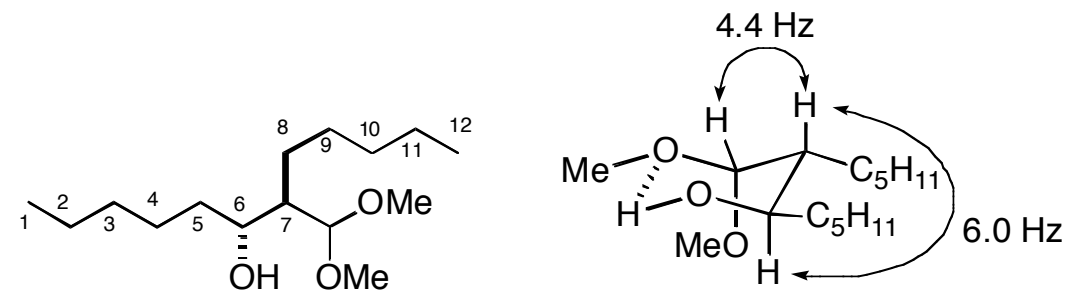

This compound was a minor product in the reaction of $\mathbf{1 a}$ with $\mathbf{2} \mathbf{b}$ and was not purely isolated. The selected signals in the crude mixture were shown below: ${ }^{1} \mathrm{H}$ NMR: $\left(270 \mathrm{MHz}, \mathrm{CDCl}_{3}\right) 4.41$ $\left(\mathrm{d}, J=4.4 \mathrm{~Hz}, 1 \mathrm{H}, 7-\mathrm{CH}(\mathrm{OMe})_{2}\right), 3.65(\mathrm{ddd}, J=8.6,6.0,2.9 \mathrm{~Hz}, 1 \mathrm{H}, 6-\mathrm{H}), 3.43\left(\mathrm{~s}, 3 \mathrm{H}, \mathrm{OMe}^{\mathrm{A}}\right)$, $3.40\left(\mathrm{~s}, 3 \mathrm{H}, \mathrm{OMe}^{\mathrm{B}}\right), 3.33\left(\mathrm{~d}, J=4.6 \mathrm{~Hz}, 1 \mathrm{H}, \mathrm{OH}, \mathrm{D}_{2} \mathrm{O}\right.$-exchangeable), 1.68-1.64 (m, 1H, 7-H); Irradiation at 7-H caused disappearance of spin-spin coupling $(J=6.0 \mathrm{~Hz})$ at $6-\mathrm{H} ;{ }^{13} \mathrm{C} \mathrm{NMR}$ : $\left(67.9 \mathrm{MHz}, \mathrm{CDCl}_{3}\right) 107.86\left(\mathrm{~d}, 13-\mathrm{CH}(\mathrm{OMe})_{2}\right), 71.21(\mathrm{~d}, \mathrm{C}-6), 55.78\left(\mathrm{q}, \mathrm{OMe}^{\mathrm{A}}\right), 54.33$ (q, $\left.\mathrm{OMe}^{\mathrm{B}}\right), 44.99(\mathrm{~d}, \mathrm{C}-7)$.

$\left(1 R^{*}, 2 S^{*}\right)-1-C y c l o h e x y l-2-d i m e t h o x y m e t h y l-1-h e p t a n o l ~(s y n-4 a c)$.
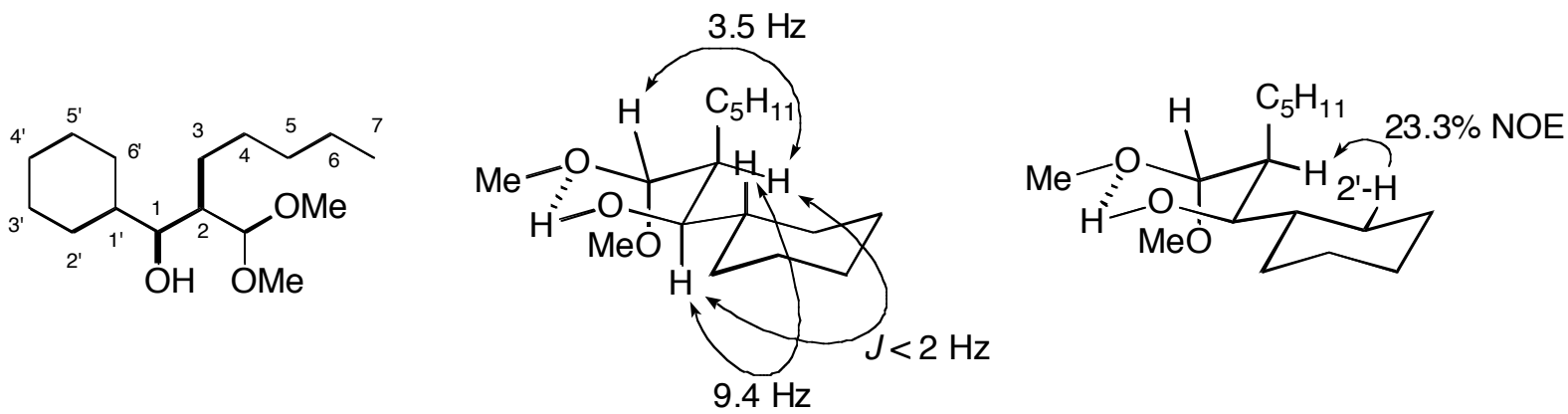

According to the general procedure, this compound was prepared from $\mathbf{1 a}, \mathbf{2 c}, \mathrm{GeCl}_{2}$-dioxane, and $\mathrm{Bu}_{4} \mathrm{NBr}$ in $\mathrm{Et}_{2} \mathrm{O}$ to give the product as a colorless liquid after flash column chromatography [hexane/ethyl acetate, 99/1 and then 95/5] on silica gel (Fuji Silysia BW-300). The syn isomer was eluted later than the anti isomer: IR: (neat) $3544(\mathrm{OH}) \mathrm{cm}^{-1} ;{ }^{1} \mathrm{H}$ NMR: $\left(600 \mathrm{MHz}, \mathrm{CDCl}_{3}\right)$ $4.35\left(\mathrm{~d}, J=3.5 \mathrm{~Hz}, 1 \mathrm{H}, 2-\mathrm{CH}(\mathrm{OMe})_{2}\right), 3.62(\mathrm{~d}, J=9.4 \mathrm{~Hz}, 1 \mathrm{H}, 1-\mathrm{H}), 3.45\left(\mathrm{~s}, 3 \mathrm{H}, \mathrm{OMe}^{\mathrm{A}}\right), 3.39$ $\left(\mathrm{s}, 3 \mathrm{H}, \mathrm{OMe}^{\mathrm{B}}\right), 2.86$ (brs, $1 \mathrm{H}, \mathrm{OH}, \mathrm{D}_{2} \mathrm{O}$-exchangeable), 2.14-2.06 (m, 1H, 6'- $\left.\mathrm{H}^{\mathrm{A}}\right), 1.78-1.69$ (m, $3 \mathrm{H}, 2-\mathrm{H}, 3^{\prime}-\mathrm{H}^{\mathrm{A}}$, and $\left.5^{\prime}-\mathrm{H}^{\mathrm{A}}\right), 1.67-1.62\left(\mathrm{~m}, 1 \mathrm{H}, 4^{\prime}-\mathrm{H}^{\mathrm{A}}\right), 1.60-1.55\left(\mathrm{~m}, 1 \mathrm{H}, 2^{\prime}-\mathrm{H}^{\mathrm{A}}\right), 1.47-1.09(\mathrm{~m}$, $12 \mathrm{H}, 3-\mathrm{H}_{2}, 4-\mathrm{H}_{2}, 5-\mathrm{H}_{2}, 6-\mathrm{H}_{2}, 1^{\prime}-\mathrm{H}, 3^{\prime}-\mathrm{H}^{\mathrm{B}}, 4^{\prime}-\mathrm{H}^{\mathrm{B}}$, and $\left.5^{\prime}-\mathrm{H}^{\mathrm{B}}\right), 0.90\left(\mathrm{t}, 3 \mathrm{H}, J=7.1 \mathrm{~Hz}, 3 \mathrm{H}, 7-\mathrm{H}_{3}\right)$,

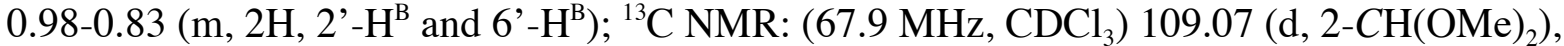
74.46 (d, C-1), 56.68 (q, OMe $\left.{ }^{\mathrm{A}}\right), 55.52\left(\mathrm{q}, \mathrm{OMe}^{\mathrm{B}}\right), 41.74(\mathrm{~d}, \mathrm{C}-2), 40.08$ (d, C-1'), 32.35 (t, C-5), 30.03 (dd, C-6'), 29.28 (t, C-2'), 28.19 (t, C-4), 26.49 (t, C-4'), 26.09 (t, C-3'), 25.96 (t, C-5'), 23.24 (t, C-3), 22.60 (t, C-6), 14.10 (q, C-7); MS: (CI, $70 \mathrm{eV}) \mathrm{m} / \mathrm{z} 255\left(\mathrm{M}^{+}-\mathrm{H}_{2} \mathrm{O}+1,3\right), 241$ $\left(\mathrm{M}^{+}-\mathrm{MeOH}+1,2\right), 223\left(\mathrm{M}^{+}-\mathrm{H}_{2} \mathrm{O}-\mathrm{MeOH}+1,16\right), 209\left(\mathrm{M}^{+}-\mathrm{MeOH}-\mathrm{OMe}, 7\right), 129$ (100), $113\left(\mathrm{C}_{6} \mathrm{H}_{11} \mathrm{CHOH}, 7\right), 95\left(\mathrm{C}_{6} \mathrm{H}_{10}=\mathrm{CH}, 3\right), 75\left(\mathrm{CH}(\mathrm{OMe})_{2}, 3\right)$; HRMS: (CI, $\left.70 \mathrm{eV}\right)$ calcd for 
$\mathrm{C}_{16} \mathrm{H}_{31} \mathrm{O}_{2} 255.2245\left(\mathrm{M}^{+}-\mathrm{H}_{2} \mathrm{O}+1\right)$ found for $\mathrm{m} / \mathrm{z}$ 255.2331. Anal. Calcd for $\mathrm{C}_{16} \mathrm{H}_{32} \mathrm{O}_{3}: \mathrm{C}, 70.54$; $\mathrm{H}, 11.84$. Found $\mathrm{C}, 70.25 ; \mathrm{H}, 11.55$.

$\left(1 R^{*}, 2 R^{*}\right)-1-C y c l o h e x y l-2-d i m e t h o x y m e t h y l-1-h e p t a n o l ~(a n t i-4 a c)$.
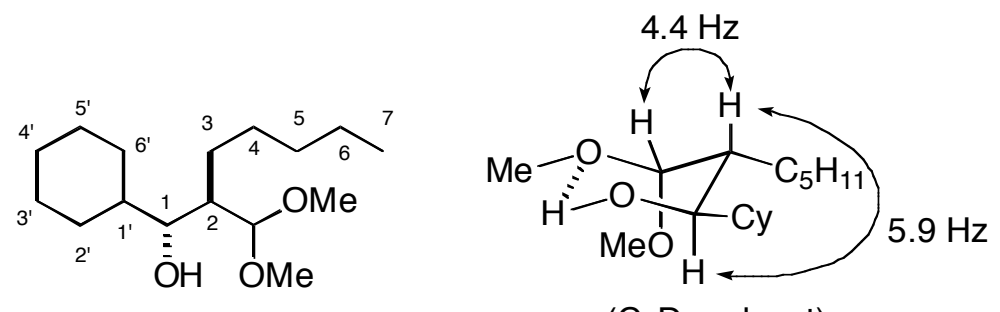

This compound was a minor product in the reaction of $\mathbf{1 a}$ with $\mathbf{2 c}$ and was not purely isolated.

The selected signals in the crude mixture were shown below: ${ }^{1} \mathrm{H}$ NMR: $\left(270 \mathrm{MHz}, \mathrm{CDCl}_{3}\right) 4.41$ $\left(\mathrm{d}, J=3.9 \mathrm{~Hz}, 1 \mathrm{H}, 2-\mathrm{CH}(\mathrm{OMe})_{2}\right), 3.43$ (s, 3H, OMe), 3.41 (s, 3H, OMe), 3.30-3.20 (brs, 1H, $\mathrm{OH}, \mathrm{D}_{2} \mathrm{O}$-exchangeable); ${ }^{1} \mathrm{H}$ NMR: $\left(270 \mathrm{MHz}, \mathrm{C}_{6} \mathrm{D}_{6}\right.$ with small amount of $\left.\mathrm{D}_{2} \mathrm{O}\right) 4.31(\mathrm{~d}, J=4.4$ $\left.\mathrm{Hz}, 1 \mathrm{H}, 2-\mathrm{CH}(\mathrm{OMe})_{2}\right), 3.57(\mathrm{dd}, J=5.9,5.9 \mathrm{~Hz}, 1 \mathrm{H}, 1-\mathrm{H}) ;{ }^{13} \mathrm{C} \mathrm{NMR}:\left(67.9 \mathrm{MHz}, \mathrm{CDCl}_{3}\right)$ $107.90\left(2-\mathrm{CH}(\mathrm{OMe})_{2}\right), 75.29(\mathrm{C}-1), 56.16(\mathrm{OMe}), 54.73(\mathrm{OMe})$.

\section{$\left(1 R^{*}, 2 R^{*}\right)$-2-Dimethoxymethyl-3-methyl-1-phenyl-1-butanol (syn-4ba).}
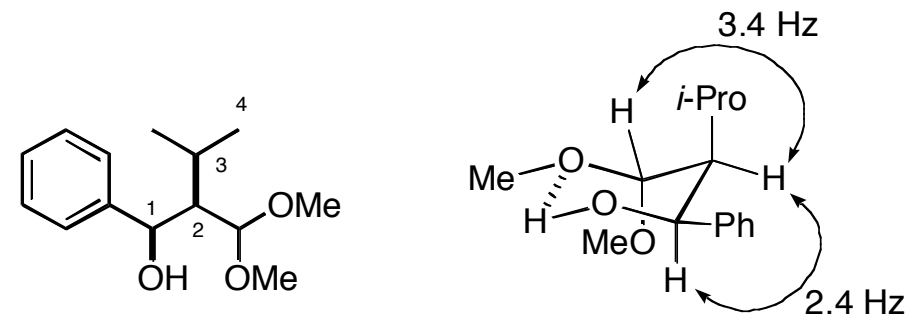

According to the general procedure, this compound was prepared from $\mathbf{1 b}, \mathbf{2} \mathbf{a}, \mathrm{GeCl}_{2}$-dioxane, and $\mathrm{Bu}_{4} \mathrm{NBr}$ in $\mathrm{Et}_{2} \mathrm{O}$ to give the product as a colorless liquid after flash column chromatography (hexane/ethyl acetate, 95/5) on silica gel (BW-300). Further purification was performed by distillation under reduced pressure: bp: $120{ }^{\circ} \mathrm{C} / 0.1 \mathrm{mmHg}$; IR: (neat) $3467(\mathrm{OH}) \mathrm{cm}^{-1}$; ${ }^{1} \mathrm{H}$ NMR: $\left(270 \mathrm{MHz}, \mathrm{CDCl}_{3}\right)$ 7.37-7.19 (m, 5H, Ar), 5.29 (brs, 1H, 1-H), 4.43 (d, J=3.4 Hz, 1H, 2$\left.\mathrm{CH}(\mathrm{OMe})_{2}\right), 3.49\left(\mathrm{~s}, 3 \mathrm{H}, \mathrm{OMe}^{\mathrm{A}}\right), 3.43\left(\mathrm{~s}, 3 \mathrm{H}, \mathrm{OMe}^{\mathrm{B}}\right), 3.29\left(\mathrm{~d}, J=2.4 \mathrm{~Hz}, 1 \mathrm{H}, \mathrm{OH}, \mathrm{D}_{2} \mathrm{O}-\right.$ exchangeable), 2.01-1.89 (m, 1H, 3-H), 1.83 (ddd, $J=3.3,3.3,3.3 \mathrm{~Hz}, 1 \mathrm{H}, 2-\mathrm{H}), 1.00$ (d, $J=7.1$ $\left.\mathrm{Hz}, 3 \mathrm{H}, 3-\mathrm{CH}_{3}\right), 0.77$ (d, $\left.J=7.1 \mathrm{~Hz}, 3 \mathrm{H}, 4-\mathrm{H}_{3}\right) ;{ }^{1} \mathrm{H} \mathrm{NMR}:\left(270 \mathrm{MHz}, \mathrm{CDCl}_{3}\right.$, small amount of $\left.\mathrm{D}_{2} \mathrm{O}\right) 7.37-7.19(\mathrm{~m}, 5 \mathrm{H}, \mathrm{Ar}), 5.29(\mathrm{~d}, J=2.4 \mathrm{~Hz} \mathrm{1H}, 1-\mathrm{H}), 4.43$ (d, $\left.J=3.4 \mathrm{~Hz}, 1 \mathrm{H}, 2-\mathrm{CH}(\mathrm{OMe})_{2}\right)$, $3.49\left(\mathrm{~s}, 3 \mathrm{H}, \mathrm{OMe}^{\mathrm{A}}\right), 3.43\left(\mathrm{~s}, 3 \mathrm{H}, \mathrm{OMe}^{\mathrm{B}}\right), 2.01-1.89(\mathrm{~m}, 1 \mathrm{H}, 3-\mathrm{H}), 1.83(\mathrm{ddd}, J=3.5,3.5,3.5 \mathrm{~Hz}$, $1 \mathrm{H}, 2-\mathrm{H}), 1.00\left(\mathrm{~d}, J=7.1 \mathrm{~Hz}, 3 \mathrm{H}, 3-\mathrm{CH}_{3}\right), 0.77\left(\mathrm{~d}, J=7.1 \mathrm{~Hz}, 3 \mathrm{H}, 4-\mathrm{H}_{3}\right) ;{ }^{13} \mathrm{C} \mathrm{NMR:}(67.9 \mathrm{MHz}$, $\left.\mathrm{CDCl}_{3}\right) 143.80(\mathrm{~s}, i-\mathrm{Ph}), 127.75(\mathrm{~d}, m-\mathrm{Ph}), 126.26(\mathrm{~d}, p-\mathrm{Ph}), 125.66(\mathrm{~d}, o-\mathrm{Ph}), 107.03(\mathrm{~d}, 2-$ $\left.\mathrm{CH}(\mathrm{OMe})_{2}\right), 72.11$ (d, C-1), 55.57 (q, OMe $\left.{ }^{\mathrm{A}}\right), 54.99$ (q, OMe $\left.{ }^{\mathrm{B}}\right), 52.25$ (d, C-2), 24.33 (d, C-3), 23.11 (q, C-4), 20.53 (q, 3- $\mathrm{CH}_{3}$ ); MS: (CI, $\left.70 \mathrm{eV}\right) \mathrm{m} / \mathrm{z} 121(\mathrm{PhCH}(\mathrm{OH}) \mathrm{CH}+1,15), 107$ $(\mathrm{PhCHOH}, 25), 101\left(\left(\mathrm{CH}_{3}\right)_{2} \mathrm{CHCHCHOMe}+1,100\right), 75\left(\mathrm{CH}(\mathrm{OMe})_{2}, 28\right)$. Anal. Calcd for $\mathrm{C}_{14} \mathrm{H}_{22} \mathrm{O}_{3}$ (238.32): C, 70.56; H, 9.30. Found C, 70.49; H, 8.87. 


\section{$\left(1 R^{*}, 2 S^{*}\right)$-2-Dimethoxymethyl-3-methyl-1-phenyl-1-butanol (anti-4ba).}<smiles>COC(OC)C(C(C)C)C(O)c1ccccc1</smiles>

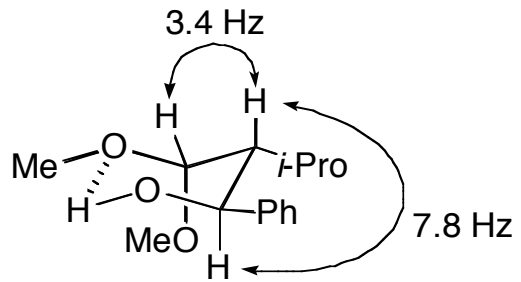

This compound was a minor product in the reaction of $\mathbf{1 b}$ with $\mathbf{2 a}$ and was not purely isolated. The selected signals in the crude mixture were shown below: ${ }^{1} \mathrm{H}$ NMR: $\left(270 \mathrm{MHz}, \mathrm{CDCl}_{3}\right) 4.93$ $(\mathrm{dd}, J=7.8,3.2 \mathrm{~Hz}, 1 \mathrm{H}, 1-\mathrm{H}), 4.41\left(\mathrm{~d}, J=3.4 \mathrm{~Hz}, 1 \mathrm{H}, 2-\mathrm{CH}(\mathrm{OMe})_{2}\right), 4.06(\mathrm{~d}, J=3.2 \mathrm{~Hz}, 1 \mathrm{H}$, $\mathrm{OH}, \mathrm{D}_{2} \mathrm{O}$-exchangeable), $3.43\left(\mathrm{~s}, 3 \mathrm{H}, \mathrm{OMe}^{\mathrm{A}}\right), 3.42\left(\mathrm{~s}, 3 \mathrm{H}, \mathrm{OMe}^{\mathrm{B}}\right), 0.95(\mathrm{~d}, J=7.1 \mathrm{~Hz}, 3 \mathrm{H}, 3-$ $\left.\mathrm{CH}_{3}\right), 0.86\left(\mathrm{~d}, J=6.8 \mathrm{~Hz}, 3 \mathrm{H}, 4-\mathrm{H}_{3}\right) ;{ }^{13} \mathrm{C} \mathrm{NMR:}\left(67.9 \mathrm{MHz}, \mathrm{CDCl}_{3}\right) 107.00\left(\mathrm{~d}, 2-\mathrm{CH}(\mathrm{OMe})_{2}\right)$, $73.41(\mathrm{~d}, \mathrm{C}-1)$.

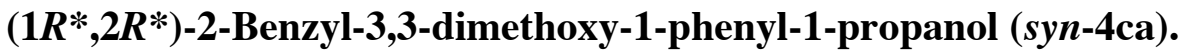<smiles>COC(OC)C(Cc1ccccc1)C(O)c1ccccc1</smiles>

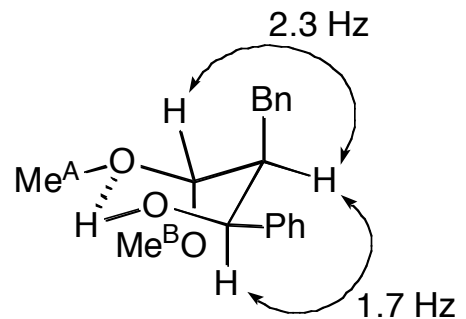

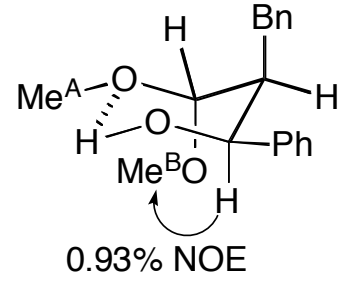

According to the general procedure, this compound was prepared from $\mathbf{1 c}, \mathbf{2 a}, \mathrm{GeCl}_{2}$-dioxane, and $\mathrm{Bu}_{4} \mathrm{NBr}$ in $\mathrm{Et}_{2} \mathrm{O}$ to give the product as a colorless liquid after flash column chromatography [hexane/ethyl acetate, 97/3 and then 95/5] on silica gel (Fuji Silysia BW-300). The syn isomer was eluted earlier than the anti isomer: IR: (neat) $3521(\mathrm{OH}) \mathrm{cm}-1 ;{ }^{1} \mathrm{H} \mathrm{NMR}$ : $\left(400 \mathrm{MHz}, \mathrm{CDCl}_{3}\right)$ 7.42-7.31 (m, 4H, 1-Ph $(o)$ and 1-Ph $(m))$, 7.26-7.16 (m, 3H, 1-Ph $(p)$ and 2- $\left.\mathrm{CH}_{2} \mathrm{Ph}(\mathrm{m})\right)$, 7.15-7.09 $\left(\mathrm{m}, 1 \mathrm{H}, 2-\mathrm{CH}_{2} P h(p)\right), 7.00-6.95\left(\mathrm{~m}, 2 \mathrm{H}, 2-\mathrm{CH}_{2} P h(o)\right), 5.31$ (brs, $\left.1 \mathrm{H}, 1-\mathrm{H}\right), 4.12(\mathrm{~d}, J=2.3 \mathrm{~Hz}$, $1 \mathrm{H}, 3-\mathrm{H}), 3.61\left(\mathrm{~d}, J=2.2 \mathrm{~Hz}, 1 \mathrm{H}, \mathrm{OH}, \mathrm{D}_{2} \mathrm{O}\right.$-exchangeable $), 3.44\left(\mathrm{~s}, 3 \mathrm{H}, \mathrm{OMe}^{\mathrm{A}}\right), 3.30(\mathrm{~s}, 3 \mathrm{H}$, $\left.\mathrm{OMe}^{\mathrm{B}}\right), 2.74\left(\mathrm{dd}, J=14.1,10.1 \mathrm{~Hz}, 1 \mathrm{H}, 2-\mathrm{CH}^{\mathrm{A}} \mathrm{Ph}\right), 2.60\left(\mathrm{dd}, J=14.1,4.5 \mathrm{~Hz}, 1 \mathrm{H}, 2-\mathrm{C} H^{\mathrm{B}} \mathrm{Ph}\right)$, 2.25-2.19 (m, 1H, 2-H); ${ }^{1} \mathrm{H}$ NMR: $\left(270 \mathrm{MHz} \mathrm{CDCl}_{3}\right.$, small amount of $\left.\mathrm{D}_{2} \mathrm{O}\right) 5.31(\mathrm{~d}, J=1.7 \mathrm{~Hz}$, 1H, 1-H); ${ }^{13} \mathrm{C}$ NMR: $\left(100 \mathrm{MHz}, \mathrm{CDCl}_{3}\right) 142.73$ (s, 1-Ph(i)), 140.47 (s, 2- $\left.\mathrm{CH}_{2} P h(i)\right)$, 128.76 (d, 2$\left.\mathrm{CH}_{2} \mathrm{Ph}(o)\right), 128.22\left(\mathrm{~d}, 2-\mathrm{CH}_{2} \mathrm{Ph}(m)\right), 127.97(\mathrm{~d}, 1-\mathrm{Ph}(m)), 126.64(\mathrm{~d}, 1-\mathrm{Ph}(p)), 125.77$ (d, 1$\mathrm{Ph}(o)), 125.75\left(\mathrm{~d}, 2-\mathrm{CH}_{2} P h(p)\right), 107.30$ (d, C-3), 71.08 (d, C-1), 56.88 (q, OMe $\left.{ }^{\mathrm{A}}\right), 55.31(\mathrm{q}$, $\mathrm{OMe}^{\mathrm{B}}$ ), 50.05 (d, C-2), 29.35 (t, 2-CH $\left.\mathrm{H}_{2} \mathrm{Ph}\right)$; MS: (EI, $\left.70 \mathrm{eV}\right) \mathrm{m} / \mathrm{z} 286\left(\mathrm{M}^{+}, 0.03\right), 254\left(\mathrm{M}^{+}-\right.$ $\mathrm{MeOH}, 0.4), 236\left(\mathrm{M}^{+}-\mathrm{H}_{2} \mathrm{O}-\mathrm{MeOH}, 0.04\right), 179\left(\mathrm{M}^{+}-\mathrm{C}_{6} \mathrm{H}_{5} \mathrm{CHOH}, 1\right), 163\left(\mathrm{M}^{+}-\mathrm{PhCH}_{2}-\right.$ $\mathrm{MeOH}, 35), 148$ (100), $147\left(\mathrm{M}^{+}-\mathrm{PhCHOH}-\mathrm{MeOH}, 39\right), 117\left(\mathrm{PhCH}_{2} \mathrm{CHCH}, 15\right), 107$ (PhCHOH, 13), $91\left(\mathrm{PhCH}_{2}, 16\right), 75\left(\mathrm{CH}(\mathrm{OMe})_{2}, 66\right)$; HRMS: (EI, $\left.70 \mathrm{eV}\right)$ calcd for $\mathrm{C}_{18} \mathrm{H}_{22} \mathrm{O}_{3}$ 
286.1569 $\left(\mathrm{M}^{+}\right)$found for $\mathrm{m} / \mathrm{z}$ 286.1557. Anal. Calcd for $\mathrm{C}_{18} \mathrm{H}_{22} \mathrm{O}_{3}: \mathrm{C}, 75.50 ; \mathrm{H}, 7.74$. Found $\mathrm{C}$, 75.41; H, 7.64.

$\left(1 R^{*}, 2 S^{*}\right)-2-B e n z y l-3,3-d i m e t h o x y-1-p h e n y l-1-p r o p a n o l ~(a n t i-4 c a)$.<smiles>COC(OC)[C@H](Cc1ccccc1)C(O)c1ccccc1</smiles>

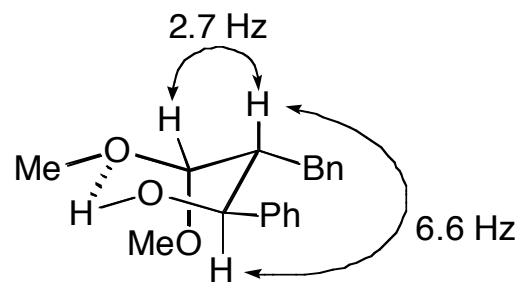

This compound was a minor product in the reaction of $\mathbf{1 c}$ with $\mathbf{2 a}$ and was not purely isolated. The selected signals in the crude mixture were shown below: ${ }^{1} \mathrm{H}$ NMR: $\left(270 \mathrm{MHz}, \mathrm{CDCl}_{3}\right) 4.78$ (dd, $J=6.6,4.4 \mathrm{~Hz}, 1 \mathrm{H}, 1-\mathrm{H}), 4.22\left(\mathrm{~d}, J=4.4 \mathrm{~Hz}, 1 \mathrm{H}, \mathrm{OH}, \mathrm{D}_{2} \mathrm{O}\right.$-exchangeable), 4.08 (d, $J=2.7$ $\mathrm{Hz}, 1 \mathrm{H}, 3-\mathrm{H}$ ), 3.38 (s, 3H, OMe), 3.36 (s, 3H, OMe); ${ }^{1} \mathrm{H}$ NMR: (270 MHz, $\mathrm{CDCl}_{3}$ with small amount of $\left.\mathrm{D}_{2} \mathrm{O}\right) 4.78(\mathrm{~d}, J=6.6 \mathrm{~Hz}, 1 \mathrm{H}, 1-\mathrm{H}) ;{ }^{13} \mathrm{C}$ NMR: $\left(67.9 \mathrm{MHz}, \mathrm{CDCl}_{3}\right) 143.06(i-\mathrm{Ph})$, 140.09 ( $i \mathrm{-Ph}), 106.99$ (C-3), $73.04(\mathrm{C}-1), 56.64(\mathrm{OMe}), 55.73$ (OMe), 49.40 (C-2), 31.84 (2$\left.\mathrm{CH}_{2} \mathrm{Ph}\right)$.

$\left(1 R^{*}, 2 R^{*}\right)$-2-Benzyl-3,3-dimethoxy-1-p-methylphenyl-1-propanol (syn-4cd).<smiles>COC(OC)C(Cc1ccccc1)C(O)c1ccc(C)cc1</smiles>

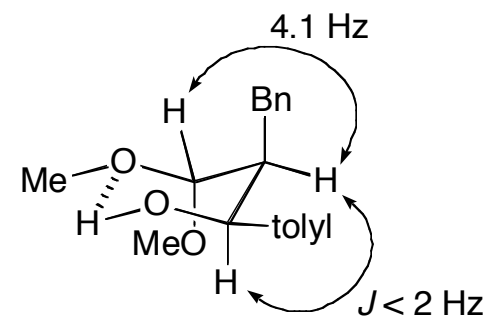

According to the general procedure, this compound was prepared from $\mathbf{1 c}, \mathbf{2 d}, \mathrm{GeCl}_{2}$-dioxane, and $\mathrm{Bu}_{4} \mathrm{NBr}$ in $\mathrm{Et}_{2} \mathrm{O}$ to give the product as a colorless liquid after flash column chromatography [hexane/ethyl acetate, 97/3 and then 95/5] on silica gel (Fuji Silysia BW-300). The syn isomer was eluted earlier than the anti isomer: IR: (neat) $3521(\mathrm{OH}) \mathrm{cm}^{-1} ;{ }^{1} \mathrm{H}$ NMR: $\left(600 \mathrm{MHz}, \mathrm{CDCl}_{3}\right)$ 7.29-7.26 (m, 2H, 2'-Hx2), 7.22-7.19 (m, 2H, 2- $\left.\mathrm{CH}_{2} P h(m)\right)$, 7.17-7.11 (m, 3H, 3'-Hx2 and 2$\left.\mathrm{CH}_{2} P h(p)\right), 7.02-6.99\left(\mathrm{~m}, 2 \mathrm{H}, 2-\mathrm{CH}_{2} P h(o)\right), 5.28-5.25$ (brs, $\left.1 \mathrm{H}, 1-\mathrm{H}\right), 4.11(\mathrm{~d}, J=4.1 \mathrm{~Hz}, 1 \mathrm{H}, 3-$ $\mathrm{H}), 3.57-3.55$ (brs, $1 \mathrm{H}, \mathrm{OH}, \mathrm{D}_{2} \mathrm{O}$-exchangeable), $3.44\left(\mathrm{~s}, 3 \mathrm{H}, \mathrm{OMe}^{\mathrm{A}}\right), 3.30\left(\mathrm{~s}, 3 \mathrm{H}, \mathrm{OMe}^{\mathrm{B}}\right), 2.74$ (dd, $\left.J=14.4,10.1 \mathrm{~Hz}, 1 \mathrm{H}, 2-\mathrm{C} H^{\mathrm{A}} \mathrm{Ph}\right), 2.60\left(\mathrm{dd}, J=14.4,4.6 \mathrm{~Hz}, 1 \mathrm{H}, 2-\mathrm{C} H^{\mathrm{B}} \mathrm{Ph}\right), 2.34$ (s, 3H, 4'$\left.\mathrm{CH}_{3}\right), 2.25-2.20(\mathrm{~m}, 1 \mathrm{H}, 2-\mathrm{H})$; ${ }^{1} \mathrm{H}$ NMR: (600 MHz, $\mathrm{CDCl}_{3}$, small amount of $\left.\mathrm{D}_{2} \mathrm{O}\right)$ 5.27-5.25 (brs, $\left.{ }^{3} J_{1-\mathrm{H},-\mathrm{H}}<2 \mathrm{~Hz}, 1 \mathrm{H}, 1-\mathrm{H}\right) ;{ }^{13} \mathrm{C}$ NMR: $\left(150 \mathrm{MHz}, \mathrm{CDCl}_{3}\right) 140.57$ (s, 2- $\left.\mathrm{CH}_{2} P h(i)\right), 139.67$ (s, C-1'), 136.11 (s, C-4'), 128.82 (d, 2- $\left.\mathrm{CH}_{2} P h(o)\right), 128.69$ (d, C-3'), 128.24 (d, 2- $\mathrm{CH}_{2} P h(m)$ ), $125.75\left(\mathrm{~d}, 2-\mathrm{CH}_{2} P h(p)\right), 125.72\left(\mathrm{~d}, \mathrm{C}-2^{\prime}\right), 107.31(\mathrm{~d}, \mathrm{C}-3), 71.08(\mathrm{~d}, \mathrm{C}-1), 56.85\left(\mathrm{q}, \mathrm{OMe}^{\mathrm{A}}\right)$, $55.23\left(\mathrm{q}, \mathrm{OMe}^{\mathrm{B}}\right), 49.99(\mathrm{~d}, \mathrm{C}-2), 29.49\left(\mathrm{t}, 2-\mathrm{CH}_{2} \mathrm{Ph}\right), 21.01$ (q, 4'-CH $)$ ) MS: (EI, $\left.70 \mathrm{eV}\right) \mathrm{m} / \mathrm{z} 300$ $\left(\mathrm{M}^{+}, 0.08\right), 268\left(\mathrm{M}^{+}-\mathrm{MeOH}, 0.4\right), 177\left(\mathrm{M}^{+}-\mathrm{PhCH}_{2}-\mathrm{MeOH}, 34\right), 148(100), 147\left(\mathrm{M}^{+}-\right.$ PhCHOH - MeOH, 24), $117\left(\mathrm{PhCH}_{2} \mathrm{CHCH}, 15\right), 91\left(\mathrm{PhCH}_{2}, 20\right), 75\left(\mathrm{CH}(\mathrm{OMe})_{2}, 44\right)$; HRMS: 
(EI, $70 \mathrm{eV}$ ) calcd for $\mathrm{C}_{19} \mathrm{H}_{24} \mathrm{O}_{3} 300.1725\left(\mathrm{M}^{+}\right)$found for $\mathrm{m} / \mathrm{z} 300.1729$. Anal. Calcd for $\mathrm{C}_{19} \mathrm{H}_{24} \mathrm{O}_{3}$ : C, 75.97; H, 8.05. Found C, 76.55; H, 8.03.

$\left(1 R^{*}, 2 S^{*}\right)-2-B e n z y l-3,3-d i m e t h o x y-1-p-m e t h y l p h e n y l-1-p r o p a n o l ~(a n t i-4 c d)$.<smiles>COC(OC)C(Cc1ccccc1)C(O)c1ccc(C)cc1</smiles>

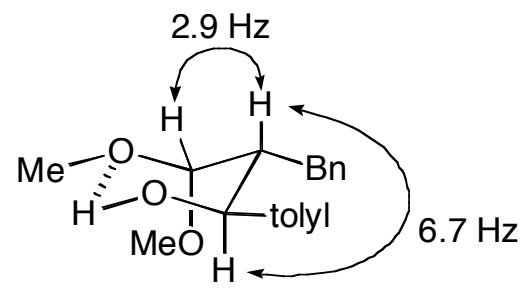

This compound was a minor product in the reaction of $\mathbf{1 c}$ with $\mathbf{2 d}$ and was not purely isolated. The selected signals in the crude mixture were shown below: ${ }^{1} \mathrm{H}$ NMR: $\left(270 \mathrm{MHz}, \mathrm{CDCl}_{3}\right)$ $4.75(\mathrm{dd}, J=6.7,4.6 \mathrm{~Hz}, 1 \mathrm{H}, 1-\mathrm{H}), 4.15\left(\mathrm{~d}, J=4.6 \mathrm{~Hz}, 1 \mathrm{H}, \mathrm{OH}, \mathrm{D}_{2} \mathrm{O}\right.$-exchangeable $), 4.09$ (d, $J=$ $2.9 \mathrm{~Hz}, 1 \mathrm{H}, 3-\mathrm{H}), 3.38$ (s, 3H, OMe), 3.35 (s, 3H, OMe), 2.35 (s, 3H, 4' $-\mathrm{CH}_{3}$ ); ${ }^{1} \mathrm{H}$ NMR: (600 $\mathrm{MHz} \mathrm{CDCl}_{3}$ with small amount of $\left.\mathrm{D}_{2} \mathrm{O}\right) 4.74(\mathrm{~d}, J=6.7 \mathrm{~Hz}, 1 \mathrm{H}, 1-\mathrm{H}) ;{ }^{13} \mathrm{C}$ NMR: $(67.9 \mathrm{MHz}$, $\left.\mathrm{CDCl}_{3}\right)$ 140.33, 140.14, 136.62, 107.14 (C-3), 73.04 (C-1), 56.68 (OMe), 55.78 (OMe), 49.50 (C-2), $32.02\left(2-\mathrm{CH}_{2} \mathrm{Ph}\right)$.

$\left(1 R^{*}, 2 R^{*}\right)-2-B e n z y l-3,3-d i m e t h o x y-1-\left(4^{\prime}\right.$-nitrophenyl)-1-propanol (syn-4ce).<smiles>COC(OC)C(Cc1ccccc1)C(O)c1ccc([N+](=O)[O-])cc1</smiles>

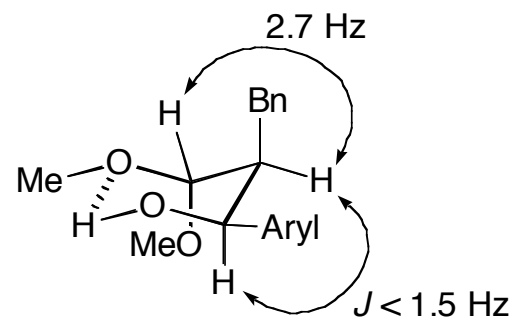

According to the general procedure, this compound was prepared from $\mathbf{1 c}, \mathbf{2 e}, \mathrm{GeCl}_{2}$-dioxane, and $\mathrm{Bu}_{4} \mathrm{NBr}$ in $\mathrm{Et}_{2} \mathrm{O}$ to give the product as a colorless liquid after flash column chromatography [hexane/ethyl acetate, 97/3 to 80/20] on silica gel (Fuji Silysia BW-300). The syn isomer was eluted earlier than the anti isomer: IR: (neat) $3514(\mathrm{OH}) \mathrm{cm}^{-1} ;{ }^{1} \mathrm{H} \mathrm{NMR:}\left(270 \mathrm{MHz}, \mathrm{CDCl}_{3}\right) 8.24-$ 8.17 (m, 2H, 3'-Hx2), 7.60-7.53 (m, 2H, 2'-Hx2), 7.25-7.10 (m, 3H, 2- $\mathrm{CH}_{2} P h(m)$ and 2$\left.\mathrm{CH}_{2} P h(p)\right)$, 6.98-6.91 (m, 2H, 2- $\left.\mathrm{CH}_{2} P h(o)\right), 5.48-5.45$ (brs, $\left.1 \mathrm{H}, 1-\mathrm{H}\right), 4.16$ (d, J = 2.7 Hz, 1H, 3$\mathrm{H}), 3.75\left(\mathrm{~d}, J=1.5 \mathrm{~Hz}, 1 \mathrm{H}, \mathrm{OH}, \mathrm{D}_{2} \mathrm{O}\right.$-exchangeable $), 3.52\left(\mathrm{~s}, 3 \mathrm{H}, \mathrm{OMe}^{\mathrm{A}}\right), 3.38\left(\mathrm{~s}, 3 \mathrm{H}, \mathrm{OMe}^{\mathrm{B}}\right)$, $2.76\left(\mathrm{dd}, J=14.4,10.6 \mathrm{~Hz}, 1 \mathrm{H}, 2-\mathrm{C} H^{\mathrm{A}} \mathrm{Ph}\right), 2.48\left(\mathrm{dd}, J=14.4,4.4 \mathrm{~Hz}, 1 \mathrm{H}, 2-\mathrm{C} H^{\mathrm{B}} \mathrm{Ph}\right.$ ), 2.25-2.16 (m, 1H, 2-H); ${ }^{1} \mathrm{H}$ NMR: (600 MHz, $\mathrm{CDCl}_{3}$, small amount of $\left.\mathrm{D}_{2} \mathrm{O}\right) 5.46-5.45$ (brs, ${ }^{3} J_{1-\mathrm{H}, 2-\mathrm{H}}<1.5$ Hz, 1H, 1-H); ${ }^{13} \mathrm{C}$ NMR: (67 MHz, $\mathrm{CDCl}_{3}$ ) 150.66 (s, C-1'), 146.49 (s, C-4'), 139.40 (s, 2$\left.\mathrm{CH}_{2} P h(i)\right), 128.52\left(\mathrm{~d}, 2-\mathrm{CH}_{2} P h(o)\right), 128.15\left(\mathrm{~d}, 2-\mathrm{CH}_{2} P h(m)\right), 126.44$ (d, C-2'), 125.83 (d, 2$\left.\mathrm{CH}_{2} P h(p)\right), 122.98$ (d, C-3'), 107.12 (d, C-3), 70.38 (d, C-1), 57.24 (q, OMe ${ }^{\mathrm{A}}$ ), 55.91 (q, $\left.\mathrm{OMe}^{\mathrm{B}}\right)$, 50.05 (d, C-2), 29.13 (t, 2- $\left.\mathrm{CH}_{2} \mathrm{Ph}\right)$; MS: (EI, $\left.70 \mathrm{eV}\right) \mathrm{m} / \mathrm{z} 331\left(\mathrm{M}^{+}, 0.5\right), 299\left(\mathrm{M}^{+}-\mathrm{MeOH}, 3\right), 282$ $\left(\mathrm{M}^{+}-\mathrm{MeO}-\mathrm{H}_{2} \mathrm{O}, 0.4\right), 208\left(\mathrm{M}^{+}-\mathrm{C}_{6} \mathrm{H}_{4} \mathrm{NO}_{2}, 16\right), 148(46), 147\left(\mathrm{M}^{+}-\mathrm{NO}_{2} \mathrm{C}_{6} \mathrm{H}_{4} \mathrm{CHOH}-\mathrm{MeOH}\right.$, 84), $133\left(\mathrm{NO}_{2} \mathrm{C}_{6} \mathrm{H}_{4}, 6\right), 117\left(\mathrm{PhCH}_{2} \mathrm{CHCH}, 14\right), 91\left(\mathrm{PhCH}_{2}, 22\right), 75\left(\mathrm{CH}(\mathrm{OMe})_{2}, 100\right)$; HRMS: 
(EI, $70 \mathrm{eV}$ ) calcd for $\mathrm{C}_{18} \mathrm{H}_{21} \mathrm{NO}_{5} 331.1420\left(\mathrm{M}^{+}\right)$found for $\mathrm{m} / \mathrm{z} 331.1422$. Anal. Calcd for $\mathrm{C}_{18} \mathrm{H}_{21} \mathrm{NO}_{5}$ : C, 65.24; H, 6.39; N, 4.23. Found C, 65.27; H, 6.42; N, 4.15.

$\left(1 R^{*}, 2 S^{*}\right)-2-B e n z y l-3,3-d i m e t h o x y-1-\left(4^{\prime}-\right.$ nitrophenyl)-1-propanol (anti-4ce).<smiles>COC(OC)[C@H](Cc1ccccc1)C(O)c1ccc([N+](=O)[O-])cc1</smiles>

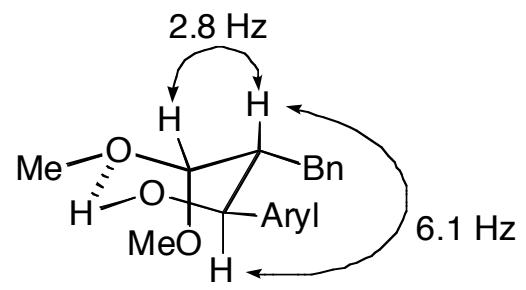

This compound was a minor product in the reaction of $\mathbf{1 c}$ with $\mathbf{2 e}$ and was not purely isolated. The selected signals in the crude mixture were shown below: ${ }^{1} \mathrm{H}$ NMR: $\left(600 \mathrm{MHz}, \mathrm{CDCl}_{3}\right)$ $4.87(\mathrm{dd}, J=6.1,5.0 \mathrm{~Hz}, 1 \mathrm{H}, 1-\mathrm{H}), 4.38\left(\mathrm{~d}, J=5.0 \mathrm{~Hz}, 1 \mathrm{H}, \mathrm{OH}, \mathrm{D}_{2} \mathrm{O}\right.$-exchangeable), 4.07 (d, $J=$ $2.8 \mathrm{~Hz}, 1 \mathrm{H}, 3-\mathrm{H}), 3.40$ (s, 3H, OMe), 3.35 (s, 3H, OMe), 2.55 (dd, J=13.9, 8.0 Hz, 1H, 2$\left.\mathrm{CH}^{\mathrm{A}} \mathrm{Ph}\right), 2.34-2.30(\mathrm{~m}, 1 \mathrm{H}, 2-\mathrm{H}) ;{ }^{1} \mathrm{H}$ NMR: $\left(600 \mathrm{MHz}, \mathrm{CDCl}_{3}\right.$ with small amount of $\left.\mathrm{D}_{2} \mathrm{O}\right)$ $4.86(\mathrm{~d}, J=6.1 \mathrm{~Hz}, 1 \mathrm{H}, 1-\mathrm{H}) ;{ }^{13} \mathrm{C}$ NMR: $\left(67.9 \mathrm{MHz}, \mathrm{CDCl}_{3}\right) 151.12$, 146.97, 139.40, 106.94 (C3), 72.58 (C-1), $56.93(\mathrm{OMe}), 55.88(\mathrm{OMe}), 49.56(\mathrm{C}-2), 32.11\left(2-\mathrm{CH}_{2} \mathrm{Ph}\right)$.

\section{$\left(2 R^{*}, 3 S^{*}\right)$-2-Benzyl-1,1-dimethoxy-3-octanol (syn-4cb).}
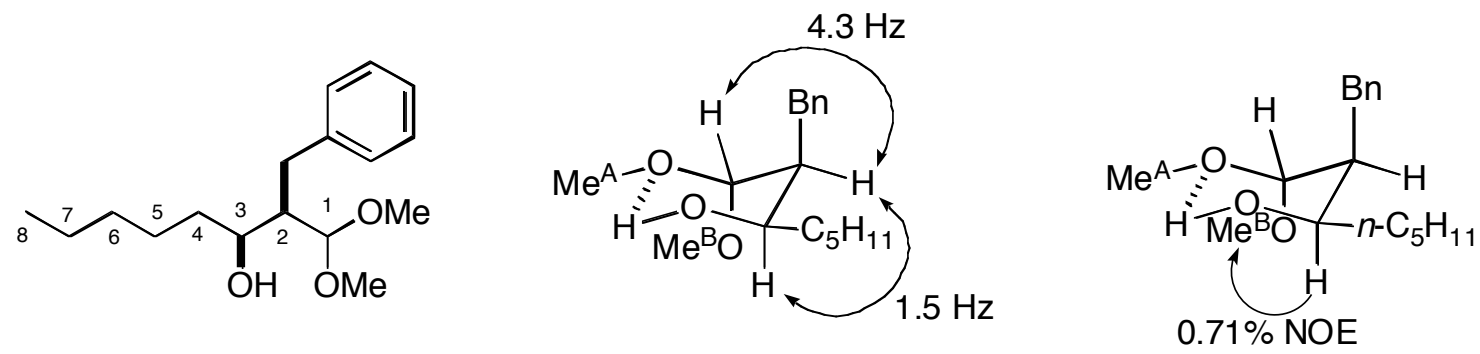

According to the general procedure, this compound was prepared from $\mathbf{1 c}, \mathbf{2 b}, \mathrm{GeCl}_{2}$-dioxane, and $\mathrm{Bu}_{4} \mathrm{NBr}$ in $\mathrm{Et}_{2} \mathrm{O}$ to give the product as a colorless liquid after flash column chromatography [hexane/ethyl acetate, 90/10 and then 80/20] on silica gel (Fuji Silysia BW-300). The product was isolated as a mixture of diastereomers: IR: (neat) $3545(\mathrm{OH}) \mathrm{cm}^{-1} ;{ }^{1} \mathrm{H}$ NMR: $(600 \mathrm{MHz}$, $\left.\mathrm{CDCl}_{3}\right)$ 7.30-7.26 (m, $\left.2 \mathrm{H}, m-\mathrm{Ph}\right), 7.22-7.17(\mathrm{~m}, 3 \mathrm{H}, o-\mathrm{Ph}$ and $p-\mathrm{Ph}), 4.18(\mathrm{~d}, J=4.3 \mathrm{~Hz}, 1 \mathrm{H}, 1-$ $\mathrm{H}), 3.97-3.93(\mathrm{~m}, 1 \mathrm{H}, 3-\mathrm{H}), 3.43\left(\mathrm{~s}, 3 \mathrm{H}, \mathrm{OMe}^{\mathrm{A}}\right), 3.28\left(\mathrm{~s}, 3 \mathrm{H}, \mathrm{OMe}^{\mathrm{B}}\right), 3.00(\mathrm{~d}, J=3.7 \mathrm{~Hz}, 1 \mathrm{H}$, $\mathrm{OH}, \mathrm{D}_{2} \mathrm{O}$-exchangeable), $2.78\left(\mathrm{dd}, J=14.5,8.7 \mathrm{~Hz}, 1 \mathrm{H}, 2-\mathrm{CH}^{\mathrm{A}} \mathrm{Ph}\right), 2.73(\mathrm{dd}, J=14.5,6.5 \mathrm{~Hz}$, 1H, 2-C $\left.H^{\mathrm{B}} \mathrm{Ph}\right), 2.06-2.01(\mathrm{~m}, 1 \mathrm{H}, 2-\mathrm{H}), 1.57-1.39\left(\mathrm{~m}, 3 \mathrm{H}, 4-\mathrm{H}_{2}\right.$ and 5- $\left.\mathrm{H}^{\mathrm{A}}\right), 1.35-1.24(\mathrm{~m}, 5 \mathrm{H}, 5-$ $\mathrm{H}^{\mathrm{B}}, 6-\mathrm{H}_{2}$ and $\left.7-\mathrm{H}_{2}\right), 0.89\left(\mathrm{t}, J=7.0 \mathrm{~Hz}, 3 \mathrm{H}, 8-\mathrm{H}_{3}\right) ;{ }^{1} \mathrm{H} \mathrm{NMR}:\left(600 \mathrm{MHz}, \mathrm{CDCl}_{3}\right.$, small amount of $\left.\mathrm{D}_{2} \mathrm{O}\right) 3.94$ (ddd, $\left.J=1.5,4.5,8.6 \mathrm{~Hz}, 1 \mathrm{H}, 3-\mathrm{H}\right)$. Irradiation at 2-H caused disappearance of spinspin coupling $(J=1.5 \mathrm{~Hz})$ at $3-\mathrm{H} .{ }^{13} \mathrm{C}$ NMR: $\left(100 \mathrm{MHz}, \mathrm{CDCl}_{3}\right) 140.75(\mathrm{~s}, i-\mathrm{Ph}), 128.87(\mathrm{~d}, o-$ $\mathrm{Ph}), 128.29(\mathrm{~d}, m-\mathrm{Ph}), 125.80(\mathrm{~d}, p-\mathrm{Ph}), 107.60(\mathrm{~d}, \mathrm{C}-1), 69.74(\mathrm{~d}, \mathrm{C}-3), 56.36\left(\mathrm{q}, \mathrm{OMe}^{\mathrm{A}}\right), 54.46$ $\left(\mathrm{q}, \mathrm{OMe}^{\mathrm{B}}\right), 47.00$ (d, C-2), 33.69 (t, C-4), 31.76 (t, C-6), 30.66 (t, 2-CH $\left.\mathrm{H}_{2} \mathrm{Ph}\right), 25.99$ (t, C-5), 22.53 (t, C-7), 13.95 (q, C-8); MS: (EI, 70 eV) m/z $280\left(\mathrm{M}^{+}, 0.06\right), 248\left(\mathrm{M}^{+}-\mathrm{MeOH}, 0.3\right), 230\left(\mathrm{M}^{+}\right.$- 
$\left.\mathrm{H}_{2} \mathrm{O}-\mathrm{MeOH}, 0.2\right), 157\left(\mathrm{M}^{+}-\mathrm{PhCH}_{2}-\mathrm{MeOH}, 3\right), 148$ (39), $147\left(\mathrm{M}^{+}-n-\mathrm{C}_{5} \mathrm{H}_{11} \mathrm{CHOH}-\mathrm{MeOH}\right.$, 39), $117\left(\mathrm{PhCH}_{2} \mathrm{CHCH}, 13\right), 91\left(\mathrm{PhCH}_{2}, 17\right), 75\left(\mathrm{CH}(\mathrm{OMe})_{2}, 100\right), 71\left(n-\mathrm{C}_{5} \mathrm{H}_{11}, 4\right)$; HRMS: (EI, $70 \mathrm{eV}$ ) calcd for $\mathrm{C}_{17} \mathrm{H}_{28} \mathrm{O}_{3} 280.2034\left(\mathrm{M}^{+}\right)$found for $\mathrm{m} / \mathrm{z} 280.2036$. Anal. Calcd for $\mathrm{C}_{17} \mathrm{H}_{28} \mathrm{O}_{3}$ 280.4024: C, 72.82; H, 10.06. Found C, 72.53; H, 9.82.

$\left(2 R^{*}, 3 R^{*}\right)$-2-Benzyl-1,1-dimethoxy-3-octanol (anti-4cb).
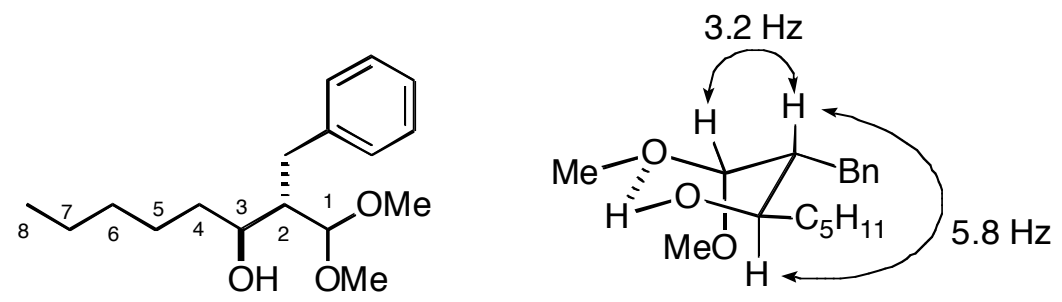

This compound was a minor product in the reaction of $\mathbf{1 c}$ with $\mathbf{2 b}$ and was not purely isolated. The selected signals in the crude mixture were shown below: ${ }^{1} \mathrm{H}$ NMR: $\left(270 \mathrm{MHz}, \mathrm{CDCl}_{3}\right)$ $4.30(\mathrm{~d}, J=3.2,1 \mathrm{H}, 1-\mathrm{H}), 3.70-3.59(\mathrm{~m}, 1 \mathrm{H}, 3-\mathrm{H}), 3.43$ (s, 3H, OMe), $3.36(\mathrm{~s}, 3 \mathrm{H}, \mathrm{OMe}) ;{ }^{1} \mathrm{H}$ NMR: $\left(600 \mathrm{MHz}, \mathrm{CDCl}_{3}\right.$ with small amount of $\left.\mathrm{D}_{2} \mathrm{O}\right) 3.63(\mathrm{td}, J=5.8,5.8 \mathrm{~Hz}, 1 \mathrm{H}, 3-\mathrm{H})$. Irradiation at 2-H caused disappearance of spin-spin coupling $(J=5.8 \mathrm{~Hz})$ at $3-\mathrm{H} .{ }^{13} \mathrm{C} \mathrm{NMR}$ : (67.9 MHz, $\left.\mathrm{CDCl}_{3}\right)$ 140.52 (i-Ph), $107.45(\mathrm{C}-1), 70.67$ (C-3), $56.30(\mathrm{OMe}), 55.34(\mathrm{OMe})$.

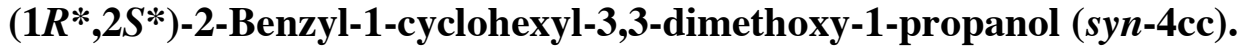
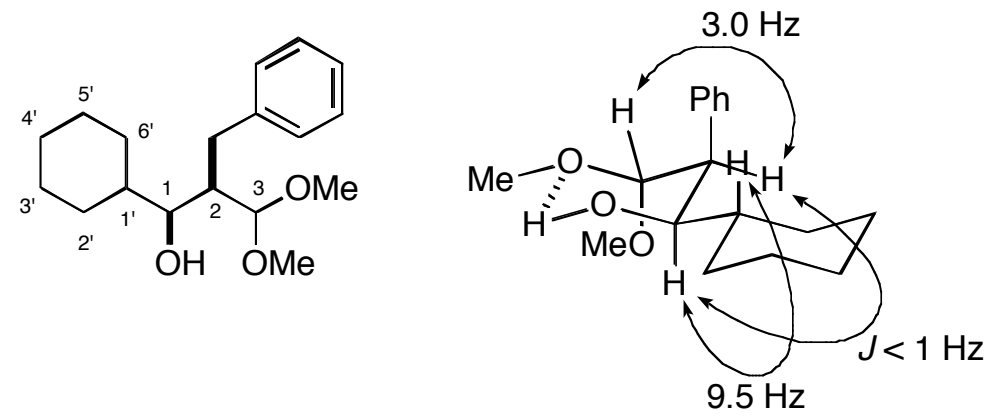

According to the general procedure, this compound was prepared from $1 \mathbf{c}, \mathbf{2 c}, \mathrm{GeCl}_{2}$-dioxane, and $\mathrm{Bu}_{4} \mathrm{NBr}$ in $\mathrm{Et}_{2} \mathrm{O}$ to give the product as a colorless liquid after flash column chromatography [hexane/ethyl acetate, 95/5] on silica gel (Fuji Silysia BW-300). The product was isolated as a mixture of diastereomers: IR: (neat) $3537(\mathrm{OH}) \mathrm{cm}^{-1} ;{ }^{1} \mathrm{H} \mathrm{NMR}:\left(600 \mathrm{MHz}, \mathrm{CDCl}_{3}\right)$ 7.30-7.25 (m, $2 \mathrm{H}, m-\mathrm{Ph}), 7.21-7.17(\mathrm{~m}, 3 \mathrm{H}, o-\mathrm{Ph}$ and $p-\mathrm{Ph}), 4.09(\mathrm{~d}, J=3.0 \mathrm{~Hz}, 1 \mathrm{H}, 3-\mathrm{H}), 3.75(\mathrm{~d}, J=9.5 \mathrm{~Hz}$, $1 \mathrm{H}, 1-\mathrm{H}), 3.41\left(\mathrm{~s}, 3 \mathrm{H}, \mathrm{OMe}^{\mathrm{A}}\right), 3.25\left(\mathrm{~s}, 3 \mathrm{H}, \mathrm{OMe}^{\mathrm{B}}\right.$ ), 3.09-3.07 (brs, 1H, OH, $\mathrm{D}_{2} \mathrm{O}$-exchangeable), $2.83\left(\mathrm{dd}, J=14.3,4.5 \mathrm{~Hz}, 1 \mathrm{H}, 2-\mathrm{C} H^{\mathrm{A}} \mathrm{Ph}\right), 2.74\left(\mathrm{dd}, J=14.3,10.1 \mathrm{~Hz}, 1 \mathrm{H}, 2-\mathrm{C} H^{\mathrm{B}} \mathrm{Ph}\right), 2.20-2.10$ $\left(\mathrm{m}, 2 \mathrm{H}, 2-\mathrm{H}\right.$ and $\left.6^{\prime}-\mathrm{H}^{\mathrm{A}}\right), 1.78-1.57\left(\mathrm{~m}, 4 \mathrm{H}, 2^{\prime}-\mathrm{H}^{\mathrm{A}}, 3^{\prime}-\mathrm{H}^{\mathrm{A}}, 4^{\prime}-\mathrm{H}^{\mathrm{A}}\right.$ and $\left.5^{\prime}-\mathrm{H}^{\mathrm{A}}\right), 1.52-1.44\left(\mathrm{~m}, 1 \mathrm{H}, 1^{\prime}-\right.$ $\mathrm{H}), 1.28-1.08\left(\mathrm{~m}, 3 \mathrm{H}, 3^{\prime}-\mathrm{H}^{\mathrm{B}}, 4^{\prime}-\mathrm{H}^{\mathrm{B}}\right.$ and $\left.5^{\prime}-\mathrm{H}^{\mathrm{B}}\right), 1.04-0.85\left(\mathrm{~m}, 2 \mathrm{H}, 2^{\prime}-\mathrm{H}^{\mathrm{B}}\right.$ and 6' $\left.-\mathrm{H}^{\mathrm{B}}\right)$. Irradiation at $1^{\prime}$-H caused disappearance of spin-spin coupling $(J=9.5 \mathrm{~Hz})$ at $1-\mathrm{H} .{ }^{13} \mathrm{C} \mathrm{NMR}$ : $(67.9 \mathrm{MHz}$, $\left.\mathrm{CDCl}_{3}\right) 140.92(\mathrm{~s}, i-\mathrm{Ph}), 128.88(\mathrm{~d}, o-\mathrm{Ph}), 128.23(\mathrm{~d}, m-\mathrm{Ph}), 125.72(\mathrm{~d}, p-\mathrm{Ph}), 108.06(\mathrm{~d}, \mathrm{C}-3)$, 73.65 (d, C-1), $56.75\left(\mathrm{q}, \mathrm{OMe}^{\mathrm{A}}\right), 55.60\left(\mathrm{q}, \mathrm{OMe}^{\mathrm{B}}\right), 43.58(\mathrm{~d}, \mathrm{C}-2), 39.85$ (d, C-1'), 30.05 (dd, C6’), 29.63 (t, 2- $\mathrm{CH}_{2} \mathrm{Ph}$ ), 29.03 (t, C-2'), 26.39 (t, C-4'), 26.00 (t, C-3'), 25.86 (t, C-5'); MS: (EI, 
$70 \mathrm{eV}) \mathrm{m} / \mathrm{z} 292\left(\mathrm{M}^{+}, 0.04\right), 260\left(\mathrm{M}^{+}-\mathrm{MeOH}, 0.3\right), 242\left(\mathrm{M}^{+}-\mathrm{H}_{2} \mathrm{O}-\mathrm{MeOH}, 0.1\right), 148$ (51), 147 $\left(\mathrm{M}^{+}-\mathrm{C}_{6} \mathrm{H}_{11} \mathrm{CHOH}-\mathrm{MeOH}, 15\right), 117\left(\mathrm{PhCH}_{2} \mathrm{CHCH}, 19\right), 91\left(\mathrm{PhCH}_{2}, 20\right), 75\left(\mathrm{CH}(\mathrm{OMe})_{2}, 100\right)$; HRMS: (EI, $70 \mathrm{eV}$ ) calcd for $\mathrm{C}_{18} \mathrm{H}_{28} \mathrm{O}_{3} 292.2038\left(\mathrm{M}^{+}\right)$found for $\mathrm{m} / \mathrm{z} 292.2044$. Anal. Calcd for $\mathrm{C}_{18} \mathrm{H}_{28} \mathrm{O}_{3} \mathrm{C}, 73.93 ; \mathrm{H}, 9.65$. Found C, 73.81; H, 9.48.

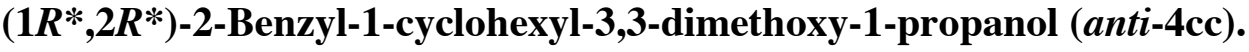
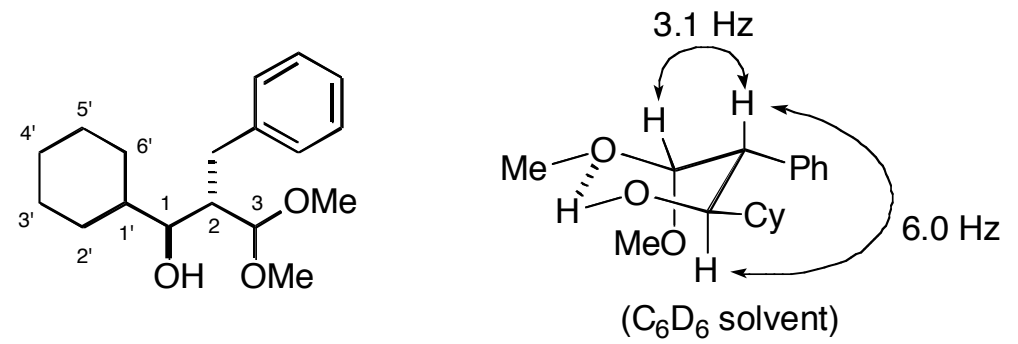

This compound was a minor product in the reaction of $\mathbf{1 c}$ with $\mathbf{2 c}$ and was not purely isolated. The selected signals in the crude mixture were shown below: ${ }^{1} \mathrm{H}$ NMR: $\left(600 \mathrm{MHz}, \mathrm{CDCl}_{3}\right) 4.31$ (d, $J=3.0 \mathrm{~Hz}, 1 \mathrm{H}, 3-\mathrm{H}), 3.44$ (s, 3H, OMe), 3.39 (s, 3H, OMe); ${ }^{1} \mathrm{H}$ NMR: $\left(600 \mathrm{MHz}, \mathrm{C}_{6} \mathrm{D}_{6}\right.$ with small amount of $\left.\mathrm{D}_{2} \mathrm{O}\right) 4.19(\mathrm{~d}, J=3.1 \mathrm{~Hz}, 1 \mathrm{H}, 3-\mathrm{H}), 3.54(\mathrm{dd}, J=6.0,6.0 \mathrm{~Hz}, 1 \mathrm{H}, 1-\mathrm{H}), 3.05(\mathrm{~s}$, 3H, OMe), 2.94 (s, 3H, OMe); ${ }^{13} \mathrm{C}$ NMR: (67.9 MHz, $\mathrm{CDCl}_{3}$ ) 140.52 (s, $\left.i-\mathrm{Ph}\right), 128.98,128.17$, 107.54 (d, C-3), 74.63 (d, C-1), 56.46 (OMe), 41.06, 31.73, 28.02, 26.29.

$\left(1 R^{*}, 2 R^{*}\right)$-2-Dimethoxymethyl-1,2,3,4-tetrahydro-1-naphthol (syn-6).

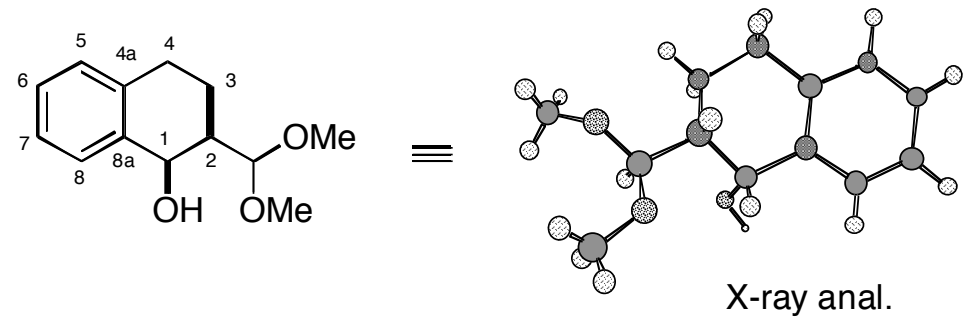

To a stirred solution of bis-aldehyde 5 ( $0.44 \mathrm{~g}$, used without purification), $\mathrm{Bu}_{4} \mathrm{NBr}(0.01 \mathrm{mmol})$, MS4A $(0.8 \mathrm{~g})$ in $\mathrm{Et}_{2} \mathrm{O}(12 \mathrm{~mL})$ was added $\mathrm{GeCl}_{2}$-dioxane $(1.1 \mathrm{mmol})$ at room temperature. After the mixture was stirred for $2 \mathrm{~h}$, at room temperature, methanol $(20 \mathrm{~mL})$ was added and the resulting solution was stirred for additional $1 \mathrm{~h}$ at room temperature. Then aqueous $\mathrm{NaHCO}_{3}$ (saturated; $50 \mathrm{~mL}$ ) was added, and the mixture was extracted with $\mathrm{Et}_{2} \mathrm{O} /$ hexane $(4 / 1)$ and the organic layer was dried over $\mathrm{MgSO}_{4}$. The solvent was evaporated under reduced pressure, and the residue was purified by flash column chromatography (hexane/ethyl acetate, 95/5) on silica gel (Fuji Silysia BW-300). The syn isomer was eluted later than anti isomer. Further purification was performed by recrystallization (hexane) to give the product as a white solid. X-ray analysis was performed and determined a stereochemistry of the compound: mp: $81{ }^{\circ} \mathrm{C}$; IR: (neat) 3413 $(\mathrm{OH}) \mathrm{cm}^{-1}$; ${ }^{1} \mathrm{H}$ NMR: $\left(600 \mathrm{MHz}, \mathrm{CDCl}_{3}\right)$ 7.34-7.31 (m, 1H, 8'-H), 7.22-7.15 (m, 2H, 6'-H, 7'-H), 7.12-7.09 (m, 1H, 5'-H), 4.81 (brs, $1 \mathrm{H}, 1-\mathrm{H}), 4.50\left(\mathrm{~d}, J=7.7 \mathrm{~Hz}, 1 \mathrm{H}, 2-\mathrm{CH}(\mathrm{OMe})_{2}\right), 3.42(\mathrm{~s}, 3 \mathrm{H}$, $\left.\mathrm{OMe}^{\mathrm{A}}\right), 3.39\left(\mathrm{~s}, 3 \mathrm{H}, \mathrm{OMe}^{\mathrm{B}}\right), 2.89\left(\mathrm{ddd}, J=17.1,5.4,2.9 \mathrm{~Hz}, 1 \mathrm{H}, 4-\mathrm{H}^{\mathrm{A}}\right), 2.76(\mathrm{ddd}, J=17.1$, 11.2, $\left.6.6 \mathrm{~Hz}, 1 \mathrm{H}, 4-\mathrm{H}^{\mathrm{B}}\right), 2.58-2.51$ (brs, $1 \mathrm{H}, \mathrm{OH}, \mathrm{D}_{2} \mathrm{O}$-exchangeable), 2.04-1.98 (m, 1H, 2-H), 
1.92-1.80 (m, 2H, 3-H $)$; ${ }^{1} \mathrm{H}$ NMR: $\left(600 \mathrm{MHz}, \mathrm{CDCl}_{3}\right.$, small amount of $\left.\mathrm{D}_{2} \mathrm{O}\right) 4.79(\mathrm{~d}, \mathrm{~J}=2.9 \mathrm{~Hz}$, 1H, 1-H); ${ }^{13} \mathrm{C}$ NMR: (150 MHz, $\left.\mathrm{CDCl}_{3}\right) 137.97$ (s, C-8a), 136.60 (s, C-4a), 129.77 (d, C-8), 128.81 (d, C-5), 127.79 (d, C-6), 126.02 (d, C-7), 105.58 (d, 2-CH(OMe) $), 67.58$ (d, C-1), 54.04 $\left(\mathrm{q}, \mathrm{OMe}^{\mathrm{A}}\right), 53.54\left(\mathrm{q}, \mathrm{OMe}^{\mathrm{B}}\right), 41.80(\mathrm{~d}, \mathrm{C}-2), 28.55(\mathrm{t}, \mathrm{C}-4), 18.64(\mathrm{t}, \mathrm{C}-3) ; \mathrm{MS}:(\mathrm{EI}, 70 \mathrm{eV}) \mathrm{m} / \mathrm{z}$ $204\left(\mathrm{M}^{+}-\mathrm{H}_{2} \mathrm{O}, 1\right), 190\left(\mathrm{M}^{+}-\mathrm{MeOH}, 1\right), 173\left(\mathrm{M}^{+}-\mathrm{H}_{2} \mathrm{O}-\mathrm{OMe}, 3\right), 130\left(\mathrm{M}^{+}-\mathrm{C}(\mathrm{OMe})_{2}-\mathrm{H}_{2} \mathrm{O}, 61\right)$, $129\left(\mathrm{M}^{+}-\mathrm{CH}(\mathrm{OMe})_{2}-\mathrm{H}_{2} \mathrm{O}, 23\right), 75\left(\mathrm{CH}(\mathrm{OMe})_{2}, 100\right), 71\left(\mathrm{M}^{+}-\mathrm{CH}(\mathrm{OMe})_{2}-\mathrm{C}_{6} \mathrm{H}_{4}, 20\right)$; HRMS: (EI, $70 \mathrm{eV}$ ) calcd for $\mathrm{C}_{13} \mathrm{H}_{16} \mathrm{O}_{2} 204.1150\left(\mathrm{M}^{+}-\mathrm{H}_{2} \mathrm{O}\right)$ found for $\mathrm{m} / \mathrm{z} 204.1153$. Anal. Calcd for $\mathrm{C}_{13} \mathrm{H}_{18} \mathrm{O}_{3}$ : C, 70.24; $\mathrm{H}, 8.16$. Found C, 70.06; H, 8.36.

$\left(1 R^{*}, 2 S^{*}\right)$-2-Dimethoxymethyl-1,2,3,4-tetrahydro-1-naphthol (anti-6).<smiles>COC(OC)C1CCc2ccccc2[C@H]1O</smiles>

This compound was a minor product in the reaction of $\mathbf{1 c}$ with $\mathbf{2 b}$ and was not purely isolated. The selected signals in the crude mixture were shown below: ${ }^{1} \mathrm{H} \mathrm{NMR}:\left(270 \mathrm{MHz}, \mathrm{CDCl}_{3}\right)$ 7.60-7.53 (m, 1H, Ar), 7.25-7.11 (m, 2H, Ar), 7.08-7.02 (m, 1H, Ar), 4.75 (d, J = 8.8 Hz, 1H, 1$\mathrm{H}), 4.40\left(\mathrm{~d}, J=6.8 \mathrm{~Hz}, 1 \mathrm{H}, 2-\mathrm{CH}(\mathrm{OMe})_{2}\right), 3.93$ (brs, $1 \mathrm{H}, \mathrm{OH}, \mathrm{D}_{2} \mathrm{O}$-exchangeable) $3.48(\mathrm{~s}, 3 \mathrm{H}$, $\left.\mathrm{OMe}^{\mathrm{A}}\right), 3.40\left(\mathrm{~s}, 3 \mathrm{H}, \mathrm{OMe}^{\mathrm{B}}\right), 2.92-2.68\left(\mathrm{~m}, 2 \mathrm{H}, 4-\mathrm{H}_{2}\right), 2.13-1.90\left(\mathrm{~m}, 2 \mathrm{H}, 2-\mathrm{H}\right.$ and $\left.3-\mathrm{H}^{\mathrm{A}}\right), 1.62-$ 1.43 (m, 1H, 3-H $\left.\left.{ }^{\mathrm{B}}\right) ;{ }^{13} \mathrm{C} \mathrm{NMR:} \mathrm{(67} \mathrm{MHz,} \mathrm{CDCl}_{3}\right) 137.94$ (s), 136.01 (s), 127.99 (d), 127.33 (d), 126.75 (d), 126.09 (d), $108.01\left(\mathrm{~d}, 2-\mathrm{CH}(\mathrm{OMe})_{2}\right), 69.71(\mathrm{~d}, \mathrm{C}-1), 55.42\left(\mathrm{q}, \mathrm{OMe}^{\mathrm{A}}\right), 52.66(\mathrm{q}$, $\mathrm{OMe}^{\mathrm{B}}$ ), 44.08 (d, C-2), 28.43 (t, C-4), 22.57 (t, C-3); MS: (EI, 70 eV) m/z $204\left(\mathrm{M}^{+}-\mathrm{H}_{2} \mathrm{O}, 2\right), 190$ $\left(\mathrm{M}^{+}-\mathrm{MeOH}, 27\right), 158\left(\mathrm{M}^{+}-2 \mathrm{MeOH}, 34\right), 130\left(\mathrm{M}^{+}-\mathrm{C}(\mathrm{OMe})_{2}-\mathrm{H}_{2} \mathrm{O}, 60\right), 129\left(\mathrm{M}^{+}-\mathrm{CH}(\mathrm{OMe})_{2}-\right.$ $\left.\mathrm{H}_{2} \mathrm{O}, 42\right), 75\left(\mathrm{CH}(\mathrm{OMe})_{2}, 100\right), 71\left(\mathrm{M}^{+}-\mathrm{CH}(\mathrm{OMe})_{2}-\mathrm{C}_{6} \mathrm{H}_{4}, 42\right)$; HRMS: (EI, $\left.70 \mathrm{eV}\right)$ calcd for $\left.\mathrm{C}_{13} \mathrm{H}_{16} \mathrm{O}_{2}\right)$ 204.1150 $\left(\mathrm{M}^{+}-\mathrm{H}_{2} \mathrm{O}\right)$ found for $\mathrm{m} / \mathrm{z} 204.1136$.

A Trial for Intramolecular Reductive Cross-Aldol Reaction of 5 Using $\mathbf{S m I}_{2}$. To a stirred solution of $\mathrm{SmI}_{2}(0.1 \mathrm{M}$ in THF, $12 \mathrm{~mL})$ was slowly added bominated bis-aldehyde $5(0.77$ $\mathrm{mmol})$ in dry THF $(5 \mathrm{~mL})$ at $-78^{\circ} \mathrm{C}$ for $10 \mathrm{~min}$. The reaction was stirred at $-78^{\circ} \mathrm{C}$ for $2 \mathrm{~h}$. A mixture of THF: $\mathrm{H}_{2} \mathrm{O}(1: 1,20 \mathrm{~mL})$ was added to the reaction mixture. The resulting mixture was extracted with $\mathrm{Et}_{2} \mathrm{O}$ /hexane (4/1), and organic layer was dried over $\mathrm{MgSO}_{4}$. The complicated mixture was observed by ${ }^{1} \mathrm{H}$ and ${ }^{13} \mathrm{C}$ NMR. The starting material 5 was totally consumed.

One-Pot Cross-Aldol Reaction for Synthesis of 4aa (Scheme 2). To a stirred solution of heptanal $7(110 \mathrm{mmol})$ and DMSO $(121 \mathrm{mmol})$ in dry acetonitrile $(100 \mathrm{~mL})$ was slowly added $\mathrm{Me}_{3} \mathrm{SiBr}(132 \mathrm{mmol})$ for $10 \mathrm{mim} .{ }^{4}$ The reaction temperature was kept at $20{ }^{\circ} \mathrm{C}$ by external cooling for $50 \mathrm{~min}$. Volatiles were removed under reduced pressure at $\mathrm{rt}$. To the resultant bromoheptanal was added dry $\mathrm{Et}_{2} \mathrm{O}(150 \mathrm{~mL})$, MS4A $(10 \mathrm{~g})$ benzaldehyde $2 \mathbf{a}(110 \mathrm{mmol})$, and $\mathrm{Bu}_{4} \mathrm{NBr}(1 \mathrm{mmol}) . \mathrm{GeCl}_{2}$-dioxane was added with keeping at $20{ }^{\circ} \mathrm{C}$ by external cooling. The reaction mixture was stirred for $2 \mathrm{~h}$ at room temperature. Methanol $(600 \mathrm{~mL})$ was added and the resulting solution was stirred for additional $1 \mathrm{~h}$ at room temperature. The mixture was slowly poured into $1 \mathrm{~L}$ of saturated $\mathrm{NaHCO}_{3}$ aq. The resulting mixture was extracted with $\mathrm{Et}_{2} \mathrm{O} /$ hexane 
(4/1), and organic layer was dried over $\mathrm{MgSO}_{4}$. The organic layer was evaporated under reduced pressure and the residue was purified by column chromatography on silica gel (BW-300, 41mm $\phi \times 45 \mathrm{~cm})$. The solvents $(1000 \mathrm{~mL}$ of hexane and $500 \mathrm{~mL}$ of hexane/EtOAc $=95 / 5)$ eluted the dimethyl acetal of benzaldehyde. The following solvents $(500 \mathrm{~mL}$ of hexane/EtOAc $=95 / 5,1000$ $\mathrm{mL}$ of hexane/EtOAc $=90 / 10$, and $500 \mathrm{~mL}$ of hexane/EtOAc $=80 / 20)$ afforded the product 4aa (17.3 g, 65\%, syn:anti $=83: 17)$.

\section{References}

(1) Roskamp, C. A.; Roskamp, E. J. Encyclopedia of Reagents for Organic Synthesis; Paquette, L. A., Ed.; John Wiley \& Sons, 1995, vol. 4, p. 2606.

(2) Duhamel, P.; Duhamel, L.; Valnot, J.-Y. Bull. Soc. Chim. Fr. 1973, 1465.

(3) Chem. Abstr. 1953, 47, 9258f.

(4) Bellesia, F.; Ghelfi, F.; Grandi, R.; Pagnoni, U. M. J. Chem. Res. (S) 1986, 428-429.

(5) Reuss, R. H.; Hassner, A. J. Org. Chem. 1974, 39, 1785-1787.

(6) Bonvallet, P. A.; Todd, E. M.; Kim, Y. S.; McMahon, R. J J. Org. Chem. 2002, 67, 90319042.

(7) Denmark, S. E.; Ghosh, S. K. Angew. Chem. Int. Ed. 2001, 40, 4759-4762.

(8) House, H. O.; Crumrine, D. S.; Teranishi, A. Y.; Olmstead, H. D. J. Am. Chem. Soc. 1973, 95, 3310-3324.

(9) Lemieux, R. U. Pure Appl. Chem. 1971, 25, 527-548. 
X-ray Structure Report for syn-6<smiles>COC(OC)C1CCc2ccccc2C1O</smiles>

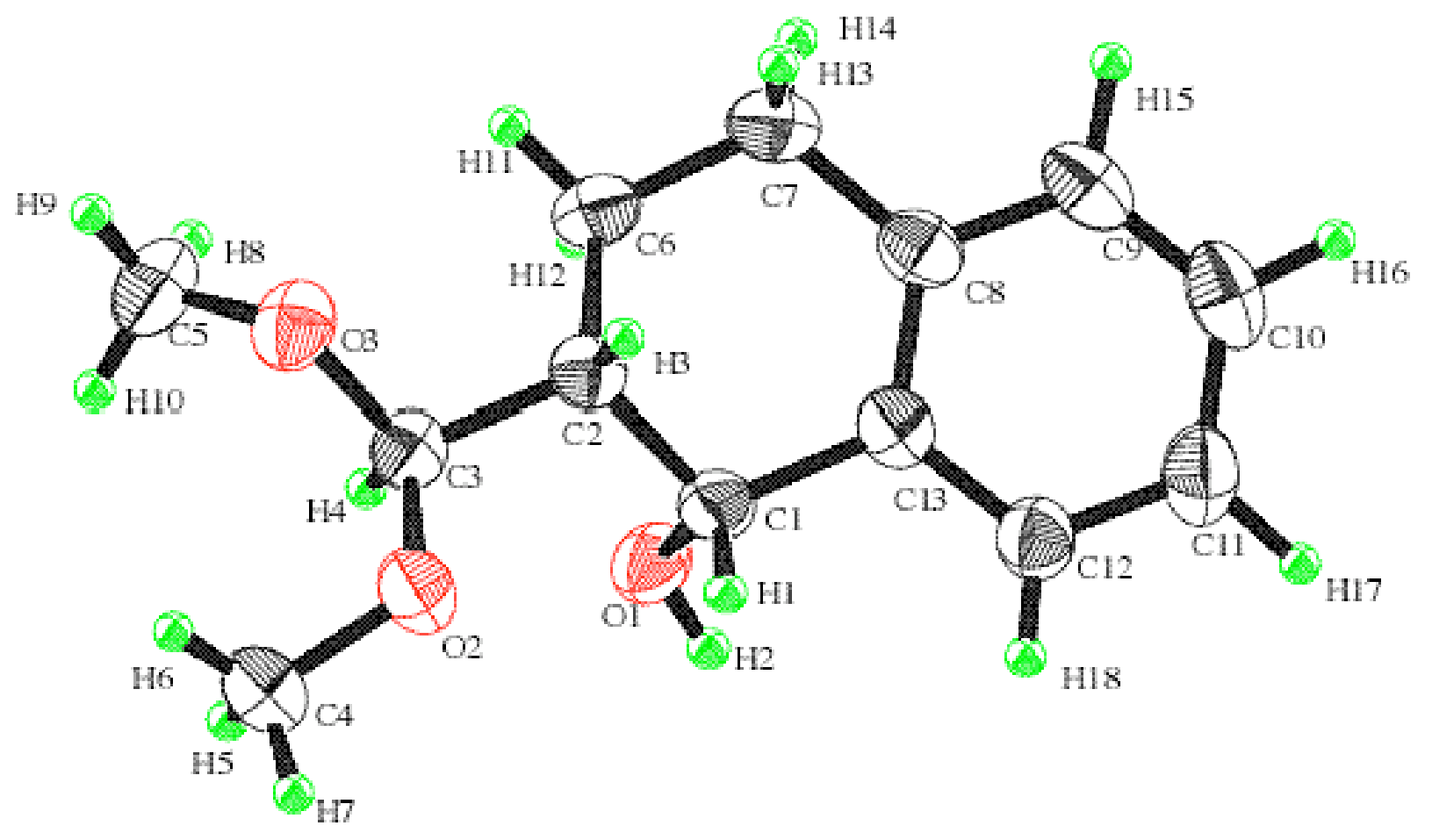




\section{Experimental}

\section{Data Collection}

A colorless block crystal of $\mathrm{C}_{13} \mathrm{H}_{18} \mathrm{O}_{3}$ having approximate dimensions of $0.50 \times 0.50 \times 0.40 \mathrm{~mm}$ was mounted on a glass fiber. All measurements were made on a Rigaku RAXIS-RAPID Imaging Plate

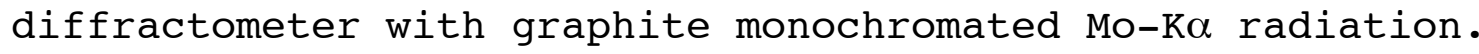

Indexing was performed from 2 oscillations which were exposed for 3.0 minutes. The camera radius was $127.40 \mathrm{~mm}$. Readout was performed in the $0.100 \mathrm{~mm}$ pixel mode.

Cell constants and an orientation matrix for data collection corresponded to a primitive monoclinic cell with dimensions:

$$
\begin{aligned}
& \mathrm{a}=7.872(1) \AA \\
& \mathrm{b}=7.923(1) \AA \\
& \mathrm{C}=9.822(1) \AA \\
& \mathrm{V}=606.4(2) \AA 3
\end{aligned}
$$

For $\mathrm{z}=2$ and F.W. $=222.28$, the calculated density is $1.22 \mathrm{~g} / \mathrm{cm}^{3}$. Based on the systematic absences of:

$0 \mathrm{k} 0: \mathrm{k} \pm 2 \mathrm{n}$

packing considerations, a statistical analysis ofintensity distribution, and the successful solution and refinement of thestructure, the space group was determined to be:

$$
\mathrm{P}_{2}(\# 4)
$$

The data were collected at a temperature of $23 \pm 1{ }^{\circ} \mathrm{C}$ to a maximum $2 \theta$ value of $54.9^{\circ}$. A total of 44 images, corresponding to $220.0 \circ$ oscillation angles, were collected with 2 different goniometer settings. Exposure time was 1.00 minutes per degree. The camera radius 
was $127.40 \mathrm{~mm}$. Readout was performed in the $0.100 \mathrm{~mm}$ pixel mode. Data were processed by the PROCESS-AUTO program package.

\section{Data Reduction}

Of the 5068 reflections which were collected, 1480 were unique $\left(R_{\text {int }}=0.026\right)$.

The linear absorption coefficient, $\mu$, for Mo-K $\alpha$ radiation is $0.9 \mathrm{~cm}^{-1}$. A symmetry-related absorption correction using the program $\mathrm{ABSCOR}^{1}$ was applied which resulted in transmission factors ranging from 0.55 to 0.97 . The data were corrected for Lorentz and polarization effects.

\section{Structure Solution and Refinement}

The structure was solved by direct methods ${ }^{2}$ and expanded using Fourier techniques ${ }^{3}$. The non-hydrogen atoms were refined anisotropically. The final cycle of full-matrix least-squares refinement ${ }^{4}$ was based on 1459 observed reflections ( $\mathrm{I}>0.00 \sigma(\mathrm{I})$, $2 \theta<54.91$ ) and 215 variable parameters and converged (largest parameter shift was 0.68 times its esd) with unweighted and weighted agreement factors of:

$$
\begin{gathered}
\mathrm{R}=\Sigma\left(\mathrm{Fo}^{2}-\mathrm{Fc}^{2}\right) / \Sigma \mathrm{Fo}^{2}=0.113 \\
\mathrm{R}_{\mathrm{W}}=\left[\left(\Sigma \mathrm{W}\left(\mathrm{FO}^{2}-\mathrm{FC}^{2}\right)^{2} / \Sigma \mathrm{W}\left(\mathrm{FO}^{2}\right)^{2}\right)\right]^{1 / 2}=0.126 \\
\mathrm{R} 1=\Sigma \| \mathrm{IFO}-\mathrm{IFClI} / \Sigma \mathrm{IFol}=0.052 \quad \text { for } \mathrm{I}>2.0 \sigma(\mathrm{I}) \text { data }
\end{gathered}
$$

The standard deviation of an observation of unit weight 5 was 2.16. The weighting scheme was based on counting statistics and included a factor $(p=0.050)$ to downweight the intense reflections. Plots of $\Sigma \mathrm{w}\left(\mathrm{FO}^{2}-\mathrm{FC}^{2}\right)^{2}$ versus $\mathrm{FO}^{2}$ reflection order in data collection, $\sin \theta / \lambda$ and various classes of indices showed no unusual trends. The maximum and minimum peaks on the final difference Fourier map corresponded to 0.29 and $-0.20 \mathrm{e}^{-/} \AA^{3}$, respectively. 
Neutral atom scattering factors were taken from Cromer and Waber 6 . Anomalous dispersion effects were included in Fcalc ${ }^{7}$; the values for $\Delta \mathrm{f}^{\prime}$ and $\Delta \mathrm{f} "$ were those of Creagh and McAuley ${ }^{8}$. The values for the mass attenuation coefficients are those of Creagh and Hubbel ${ }^{9}$. All calculations were performed using the texsan ${ }^{10}$ crystallographic software package of Molecular Structure Corporation.

\section{References}

(1) ABSCOR: Higashi T. (1995). Program for Absorption Correction, Rigaku Corporation, Tokyo, Japan.

(2) SHELXS-97: Sheldrick, G.M. (1997). Program for the Solution of Crystal Structuers. University of Goettingen, Germany.

(3) DIRDIF94: Beurskens, P.T., Admiraal, G., Beurskens, G., Bosman, W.P., de Gelder, R., Israel, R. and Smits, J.M.M. (1994). The DIRDIF-94 program system, Technical Report of the Crystallography Laboratory, University of Nijmegen, The Netherlands.

(4) Least-Squares :

Function minimized: $\quad \sum w\left(\mathrm{~F}_{\mathrm{O}}^{2}-\mathrm{F}_{\mathrm{C}}{ }^{2}\right)^{2}$ where

$\left.\left.\left(\operatorname{Max}\left(\mathrm{FO}^{2}, 0\right)+2 \mathrm{FC}^{2}\right) / 3\right)^{2}\right]^{-1}$

$$
w=1 /\left[\sigma^{2}\left(\mathrm{FO}^{2}\right)\right]=\left[\sigma_{\mathrm{C}}^{2}\left(\mathrm{FO}^{2}\right)+(\mathrm{p}\right.
$$

$\sigma_{\mathrm{C}}\left(\mathrm{FO}^{2}\right)=$ e.s.d. based on counting statistics

$p=p$-factor

(5) Standard deviation of an observation of unit weight:

$$
\begin{aligned}
& {\left[\sum w\left(\left|F_{O}\right|-\left|F_{C}\right|\right)^{2} /\left(N_{O}-N_{V}\right)\right]^{1 / 2}} \\
& \text { where: } N_{O}=\text { number of observations } \\
& \quad N_{V}=\text { number of variables }
\end{aligned}
$$

(6) Cromer, D. T. \& Waber, J. T.; "International Tables for X-ray Crystallography", Vol. IV, The Kynoch Press, Birmingham, England, 
Table 2.2 A (1974).

(7) Ibers, J.A. \& Hamilton, W. C. ; Acta Crystallogr ., 17, 781 (1964) .

(8) Creagh, D. C. \& McAuley, W.J .; "International Tables for Crystallography", Vol C, (A.J.C. Wilson, ed.), Kluwer Academic Publishers, Boston, Table 4.2.6.8, pages 219-222 (1992).

(9) Creagh, D. C. \& Hubbell, J.H..; "International Tables for Crystallography", Vol C, (A.J.C. Wilson, ed.), Kluwer Academic Publishers, Boston, Table 4.2.4.3, pages 200-206 (1992).

(10) texsan: Crystal Structure Analysis Package, Molecular Structure Corporation (1985\&1999). 


\section{A. Crystal Data}

Empirical Formula

Formula Weight

Crystal Color, Habit

Crystal Dimensions

Crystal system

Lattice Type

No. of Reflections Used for Unit

Cell Determination ( $2 \theta$ range)

Indexing Images

Camera Radius

Lattice Parameters
$\mathrm{C}_{13} \mathrm{H}_{18} \mathrm{O}_{3}$

222.28

colorless, block

$0.50 \times 0.50 \times 0.40 \mathrm{~mm}$

monoclinic

Primitive

$6040\left(4.2-54.8^{\circ}\right)$

2 oscillations at 3.0 minutes

$127.40 \mathrm{~mm}$

$\mathrm{a}=7.872(1) \AA$

$\mathrm{b}=7.923(1) \AA$

$\mathrm{C}=9.822(1) \AA$

$\beta=98.156(9)^{\circ}$

$\mathrm{V}=606.4(2) \AA^{3}$

Space Group

P2 1 (\#4)

2

$1.217 \mathrm{~g} / \mathrm{cm}^{3}$

240.00

$0.85 \mathrm{~cm}^{-1}$

B. Intensity Measurements

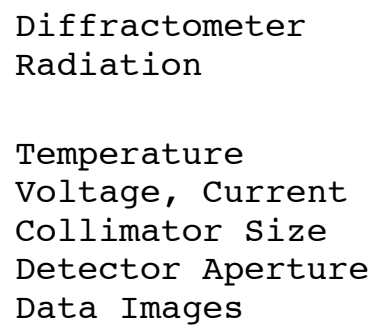

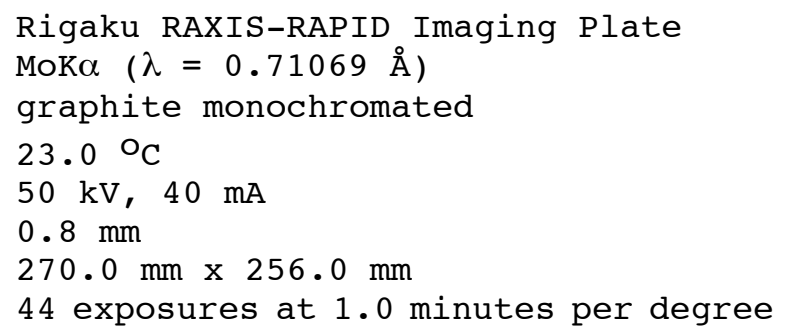




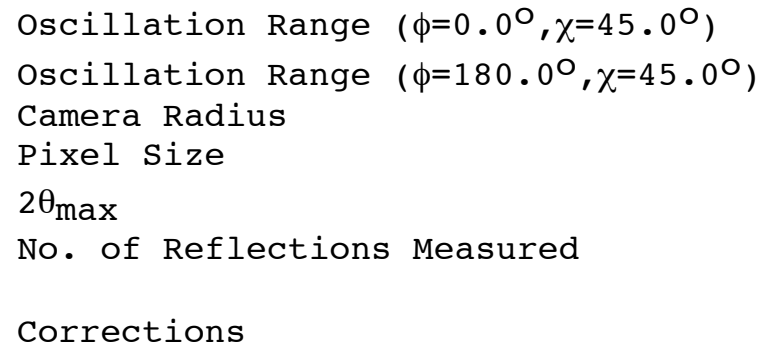

\section{Structure Solution and Refinement}

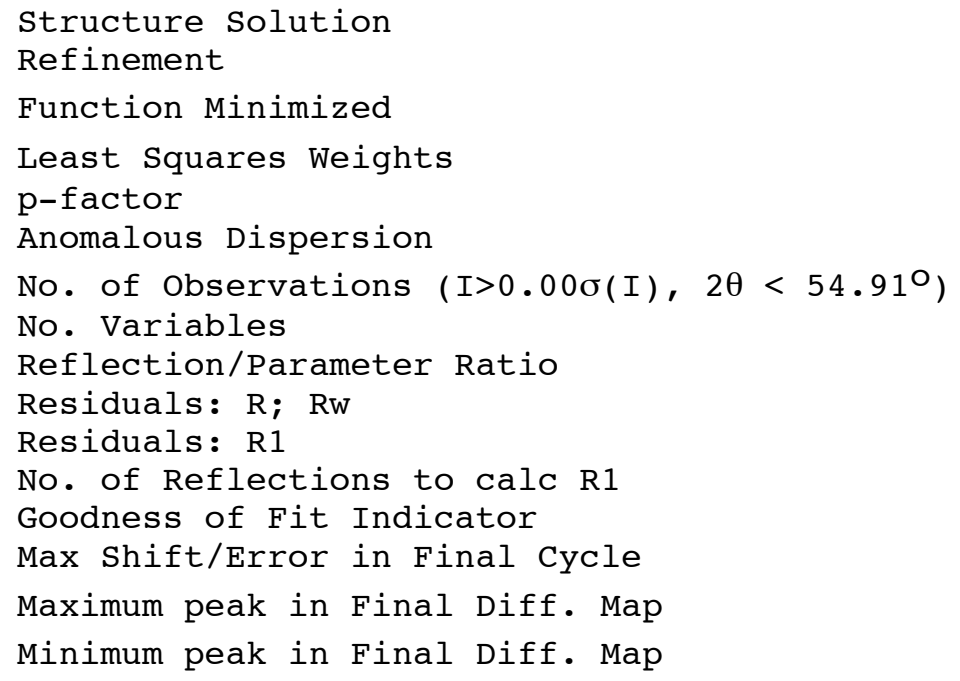


Table 1. Atomic coordinates and $\mathrm{B}_{\text {iso }} / \mathrm{B}_{\text {eq }}$

\begin{tabular}{|c|c|c|c|c|}
\hline atom & $\mathrm{x}$ & $y$ & $\mathrm{z}$ & $\mathrm{B}_{\text {eq }}$ \\
\hline$O(1)$ & $0.0729(2)$ & 0.8711 & $0.3531(1)$ & $4.33(2)$ \\
\hline$O(2)$ & $0.1680(2)$ & 0.6294 & $0.6338(1)$ & $4.44(2)$ \\
\hline$O(3)$ & $0.4180(2)$ & $0.5374(2)$ & $0.5622(1)$ & 4.69 ( 3 ) \\
\hline$C(1)$ & $0.0117(2)$ & $0.6996(3)$ & $0.3583(1)$ & $3.54(3)$ \\
\hline$C(2)$ & $0.1686(2)$ & $0.5867(3)$ & $0.3950(1)$ & $3.60(3)$ \\
\hline$C(3)$ & $0.2740(2)$ & $0.6428(2)$ & $0.5296(2)$ & $3.67(3)$ \\
\hline$C(4)$ & $0.2399(3)$ & $0.6964(4)$ & $0.7639(2)$ & $5.49(5)$ \\
\hline$C(5)$ & $0.5780(3)$ & $0.6211(4)$ & $0.5762(3)$ & $5.63(5)$ \\
\hline$C(6)$ & $0.2709(2)$ & $0.5846(3)$ & $0.2738(2)$ & $4.35(3)$ \\
\hline$C(7)$ & 0.1666 (3) & $0.4973(4)$ & $0.1511(2)$ & $5.15(4)$ \\
\hline$C(8)$ & $-0.0161(2)$ & $0.5570(3)$ & $0.1234(2)$ & $4.06(3)$ \\
\hline$C(9)$ & $-0.1184(3)$ & $0.5138(3)$ & $-0.0003(2)$ & $4.93(4)$ \\
\hline$C(10)$ & $-0.2874(3)$ & $0.5605(3)$ & $-0.0266(2)$ & $5.51(4)$ \\
\hline$C(11)$ & $-0.3611(2)$ & 0.6555 (3) & $0.0700(2)$ & $5.31(4)$ \\
\hline$C(12)$ & $-0.2621(2)$ & $0.6986(3)$ & $0.1930(2)$ & $4.33(3)$ \\
\hline$C(13)$ & $-0.0907(2)$ & $0.6501(3)$ & $0.2209(1)$ & $3.72(3)$ \\
\hline $\mathrm{H}(1)$ & $-0.059(2)$ & $0.687(3)$ & $0.423(2)$ & $3.2(3)$ \\
\hline $\mathrm{H}(2)$ & $-0.010(3)$ & $0.937(3)$ & $0.349(2)$ & $4.8(4)$ \\
\hline $\mathrm{H}(3)$ & $0.131(2)$ & $0.478(3)$ & $0.403(2)$ & $2.6(3)$ \\
\hline $\mathrm{H}(4)$ & $0.316(2)$ & $0.757(3)$ & $0.526(2)$ & $3.1(3)$ \\
\hline $\mathrm{H}(5)$ & $0.282(4)$ & $0.811(5)$ & $0.751(3)$ & $6.6(6)$ \\
\hline $\mathrm{H}(6)$ & $0.333(3)$ & $0.632(4)$ & $0.808(3)$ & $6.1(5)$ \\
\hline $\mathrm{H}(7)$ & $0.145(4)$ & $0.690(4)$ & $0.825(3)$ & 6.9 ( 6 ) \\
\hline $\mathrm{H}(8)$ & $0.596(3)$ & $0.670(4)$ & $0.495(3)$ & $7.3(7)$ \\
\hline $\mathrm{H}(9)$ & $0.655(5)$ & $0.541(5)$ & $0.600(4)$ & $8.6(8)$ \\
\hline $\mathrm{H}(10)$ & $0.581(4)$ & $0.706(5)$ & $0.644(3)$ & 7.7 \\
\hline $\mathrm{H}(11)$ & $0.381(3)$ & $0.528(3)$ & $0.292(2)$ & $5.0(5)$ \\
\hline $\mathrm{H}(12)$ & $0.302(3)$ & $0.701(3)$ & $0.248(2)$ & $4.0(4)$ \\
\hline $\mathrm{H}(13)$ & 0.168 (3) & $0.376(5)$ & $0.165(3)$ & $6.4(6)$ \\
\hline $\mathrm{H}(14)$ & $0.212(3)$ & $0.512(5)$ & $0.067(3)$ & $7.3(7)$ \\
\hline $\mathrm{H}(15)$ & $-0.073(3)$ & $0.441(4)$ & $-0.069(3)$ & $6.7(6)$ \\
\hline $\mathrm{H}(16)$ & $-0.350(3)$ & $0.515(3)$ & $-0.111(2)$ & $5.6(5)$ \\
\hline $\mathrm{H}(17)$ & $-0.487(3)$ & $0.700(4)$ & $0.053(2)$ & $6.1(5)$ \\
\hline $\mathrm{H}(18)$ & $-0.316(3)$ & $0.762(3)$ & $0.254(2)$ & $4.6(4)$ \\
\hline
\end{tabular}


Table 2. Anisotropic Displacement Parameters

\begin{tabular}{llllrrr} 
atom & \multicolumn{1}{c}{$\mathrm{U}_{11}$} & \multicolumn{1}{c}{$\mathrm{U}_{22}$} & \multicolumn{1}{l}{$\mathrm{U}_{33}$} & \multicolumn{1}{c}{$\mathrm{U}_{12}$} & \multicolumn{1}{c}{$\mathrm{U}_{13}$} & \multicolumn{1}{c}{$\mathrm{U}_{23}$} \\
$\mathrm{O}(1)$ & $0.0457(6)$ & $0.0443(6)$ & $0.0728(7)$ & $0.0025(5)$ & $0.0030(5)$ & $-0.0048(5)$ \\
$\mathrm{O}(2)$ & $0.0583(6)$ & $0.0634(8)$ & $0.0465(5)$ & $-0.0113(6)$ & $0.0060(4)$ & $-0.0060(5)$ \\
$\mathrm{O}(3)$ & $0.0576(6)$ & $0.0434(6)$ & $0.0741(7)$ & $0.0071(5)$ & $-0.0014(5)$ & $0.0060(5)$ \\
$\mathrm{C}(1)$ & $0.0407(6)$ & $0.0509(7)$ & $0.0445(6)$ & $-0.0035(6)$ & $0.0110(5)$ & $-0.0043(6)$ \\
$\mathrm{C}(2)$ & $0.0482(7)$ & $0.0410(7)$ & $0.0482(6)$ & $-0.0027(6)$ & $0.0086(5)$ & $-0.0004(6)$ \\
$\mathrm{C}(3)$ & $0.0460(7)$ & $0.0383(7)$ & $0.0549(7)$ & $-0.0031(6)$ & $0.0062(5)$ & $0.0011(6)$ \\
$\mathrm{C}(4)$ & $0.074(1)$ & $0.076(1)$ & $0.0571(9)$ & $0.001(1)$ & $0.0022(8)$ & $-0.0175(9)$ \\
$\mathrm{C}(5)$ & $0.0487(9)$ & $0.074(1)$ & $0.089(1)$ & $0.0072(8)$ & $0.0011(9)$ & $0.006(1)$ \\
$\mathrm{C}(6)$ & $0.0506(8)$ & $0.0593(10)$ & $0.0583(8)$ & $0.0078(8)$ & $0.0178(6)$ & $0.0004(7)$ \\
$\mathrm{C}(7)$ & $0.0681(10)$ & $0.074(1)$ & $0.0565(9)$ & $0.0112(9)$ & $0.0177(7)$ & $-0.0112(8)$ \\
$\mathrm{C}(8)$ & $0.0631(8)$ & $0.0492(9)$ & $0.0438(6)$ & $-0.0057(7)$ & $0.0136(5)$ & $-0.0007(6)$ \\
$\mathrm{C}(9)$ & $0.085(1)$ & $0.062(1)$ & $0.0415(7)$ & $-0.0094(9)$ & $0.0112(7)$ & $-0.0063(7)$ \\
$\mathrm{C}(10)$ & $0.082(1)$ & $0.078(1)$ & $0.0443(8)$ & $-0.018(1)$ & $-0.0070(7)$ & $0.0031(8)$ \\
$\mathrm{C}(11)$ & $0.0573(9)$ & $0.084(1)$ & $0.0565(8)$ & $-0.0058(10)$ & $-0.0043(6)$ & $0.0054(8)$ \\
$\mathrm{C}(12)$ & $0.0499(7)$ & $0.063(1)$ & $0.0513(7)$ & $-0.0042(8)$ & $0.0067(6)$ & $-0.0011(8)$ \\
$\mathrm{C}(13)$ & $0.0509(7)$ & $0.0478(8)$ & $0.0433(6)$ & $-0.0080(7)$ & $0.0094(5)$ & $0.0005(6)$
\end{tabular}

The general temperature factor expression:

$\exp \left(-2 \pi^{2}\left(a * 2 U_{11} h^{2}+b * 2 U_{22} k^{2}+c * 2 U_{33} l^{2}+2 a * b * U_{12} h k+2 a * c * U_{13} h l+\right.\right.$ $\left.2 \mathrm{~b} * \mathrm{C} * \mathrm{U}_{23} \mathrm{kl}\right)$ )

Table 3. Bond Lengths $(\AA)$

$\begin{array}{llllll}\text { atom } & \text { atom } & \text { distance } & \text { atom } & \text { atom } & \text { distance } \\ & & & & \\ \mathrm{O}(1) & \mathrm{C}(1) & 1.445(3) & \mathrm{O}(2) & \mathrm{C}(3) & 1.414(3) \\ \mathrm{O}(2) & \mathrm{C}(4) & 1.424(3) & \mathrm{O}(3) & \mathrm{C}(3) & 1.408(3) \\ \mathrm{O}(3) & \mathrm{C}(5) & 1.413(4) & \mathrm{C}(1) & \mathrm{C}(2) & 1.526(3) \\ \mathrm{C}(1) & \mathrm{C}(13) & 1.522(3) & \mathrm{C}(2) & \mathrm{C}(3) & 1.524(3) \\ \mathrm{C}(2) & \mathrm{C}(6) & 1.529(3) & \mathrm{C}(6) & \mathrm{C}(7) & 1.524(4) \\ \mathrm{C}(7) & \mathrm{C}(8) & 1.502(4) & \mathrm{C}(8) & \mathrm{C}(9) & 1.402(4) \\ \mathrm{C}(8) & \mathrm{C}(13) & 1.401(3) & \mathrm{C}(9) & \mathrm{C}(10) & 1.370(5) \\ \mathrm{C}(10) & \mathrm{C}(11) & 1.400(5) & \mathrm{C}(11) & \mathrm{C}(12) & 1.384(4) \\ \mathrm{C}(12) & \mathrm{C}(13) & 1.392(4) & & & \end{array}$


Table 4. Bond Lengths ( $\AA$ )

$\begin{array}{llllll}\text { atom } & \text { atom } & \text { distance } & \text { atom } & \text { atom } & \text { distance } \\ & & & & & \\ \mathrm{O}(1) & \mathrm{H}(2) & 0.83(4) & \mathrm{C}(1) & \mathrm{H}(1) & 0.91(3) \\ \mathrm{C}(2) & \mathrm{H}(3) & 0.92(3) & \mathrm{C}(3) & \mathrm{H}(4) & 0.96(3) \\ \mathrm{C}(4) & \mathrm{H}(5) & 0.98(5) & \mathrm{C}(4) & \mathrm{H}(6) & 0.95(4) \\ \mathrm{C}(4) & \mathrm{H}(7) & 1.02(4) & \mathrm{C}(5) & \mathrm{H}(8) & 0.91(5) \\ \mathrm{C}(5) & \mathrm{H}(9) & 0.89(6) & \mathrm{C}(5) & \mathrm{H}(10) & 0.95(6) \\ \mathrm{C}(6) & \mathrm{H}(11) & 0.97(3) & \mathrm{C}(6) & \mathrm{H}(12) & 1.00(4) \\ \mathrm{C}(7) & \mathrm{H}(13) & 0.97(5) & \mathrm{C}(7) & \mathrm{H}(14) & 0.95(5) \\ \mathrm{C}(9) & \mathrm{H}(15) & 0.99(4) & \mathrm{C}(10) & \mathrm{H}(16) & 0.97(4) \\ \mathrm{C}(11) & \mathrm{H}(17) & 1.05(4) & \mathrm{C}(12) & \mathrm{H}(18) & 0.93(4)\end{array}$

Table 5. Bond Angles ( $\left.{ }^{\circ}\right)$

$\begin{array}{llllllll}\text { atom } & \text { atom } & \text { atom } & \text { angle } & \text { atom } & \text { atom } & \text { atom } & \text { angle } \\ \mathrm{C}(3) & \mathrm{O}(2) & \mathrm{C}(4) & 114.9(2) & \mathrm{C}(3) & \mathrm{O}(3) & \mathrm{C}(5) & 114.9(2) \\ \mathrm{O}(1) & \mathrm{C}(1) & \mathrm{C}(2) & 107.3(2) & \mathrm{O}(1) & \mathrm{C}(1) & \mathrm{C}(13) & 110.4(2) \\ \mathrm{C}(2) & \mathrm{C}(1) & \mathrm{C}(13) & 111.6(2) & \mathrm{C}(1) & \mathrm{C}(2) & \mathrm{C}(3) & 110.9(2) \\ \mathrm{C}(1) & \mathrm{C}(2) & \mathrm{C}(6) & 108.7(2) & \mathrm{C}(3) & \mathrm{C}(2) & \mathrm{C}(6) & 113.3(2) \\ \mathrm{O}(2) & \mathrm{C}(3) & \mathrm{O}(3) & 109.2(2) & \mathrm{O}(2) & \mathrm{C}(3) & \mathrm{C}(2) & 107.6(2) \\ \mathrm{O}(3) & \mathrm{C}(3) & \mathrm{C}(2) & 110.3(2) & \mathrm{C}(2) & \mathrm{C}(6) & \mathrm{C}(7) & 109.6(2) \\ \mathrm{C}(6) & \mathrm{C}(7) & \mathrm{C}(8) & 113.3(2) & \mathrm{C}(7) & \mathrm{C}(8) & \mathrm{C}(9) & 119.8(2) \\ \mathrm{C}(7) & \mathrm{C}(8) & \mathrm{C}(13) & 121.7(2) & \mathrm{C}(9) & \mathrm{C}(8) & \mathrm{C}(13) & 118.4(2) \\ \mathrm{C}(8) & \mathrm{C}(9) & \mathrm{C}(10) & 121.4(3) & \mathrm{C}(9) & \mathrm{C}(10) & \mathrm{C}(11) & 120.1(2) \\ \mathrm{C}(10) & \mathrm{C}(11) & \mathrm{C}(12) & 119.2(3) & \mathrm{C}(11) & \mathrm{C}(12) & \mathrm{C}(13) & 120.9(3) \\ \mathrm{C}(1) & \mathrm{C}(13) & \mathrm{C}(8) & 121.4(2) & \mathrm{C}(1) & \mathrm{C}(13) & \mathrm{C}(12) & 118.7(2) \\ \mathrm{C}(8) & \mathrm{C}(13) & \mathrm{C}(12) & 119.9(2) & & & & \end{array}$

Table 6. Bond Angles ( $\left.{ }^{\circ}\right)$

$\begin{array}{llllllll}\text { atom } & \text { atom } & \text { atom } & \text { angle } & \text { atom } & \text { atom } & \text { atom } & \text { angle } \\ \mathrm{C}(1) & \mathrm{O}(1) & \mathrm{H}(2) & 109(2) & \mathrm{O}(1) & \mathrm{C}(1) & \mathrm{H}(1) & 111(1) \\ \mathrm{C}(2) & \mathrm{C}(1) & \mathrm{H}(1) & 108(1) & \mathrm{C}(13) & \mathrm{C}(1) & \mathrm{H}(1) & 107(1) \\ \mathrm{C}(1) & \mathrm{C}(2) & \mathrm{H}(3) & 108(1) & \mathrm{C}(3) & \mathrm{C}(2) & \mathrm{H}(3) & 109(1)\end{array}$




$\begin{array}{llllllll}\mathrm{C}(6) & \mathrm{C}(2) & \mathrm{H}(3) & 105(1) & \mathrm{O}(2) & \mathrm{C}(3) & \mathrm{H}(4) & 109(1) \\ \mathrm{O}(3) & \mathrm{C}(3) & \mathrm{H}(4) & 107(1) & \mathrm{C}(2) & \mathrm{C}(3) & \mathrm{H}(4) & 112(1) \\ \mathrm{O}(2) & \mathrm{C}(4) & \mathrm{H}(5) & 109(2) & \mathrm{O}(2) & \mathrm{C}(4) & \mathrm{H}(6) & 113(2) \\ \mathrm{O}(2) & \mathrm{C}(4) & \mathrm{H}(7) & 106(2) & \mathrm{H}(5) & \mathrm{C}(4) & \mathrm{H}(6) & 107(3) \\ \mathrm{H}(5) & \mathrm{C}(4) & \mathrm{H}(7) & 113(4) & \mathrm{H}(6) & \mathrm{C}(4) & \mathrm{H}(7) & 106(3) \\ \mathrm{O}(3) & \mathrm{C}(5) & \mathrm{H}(8) & 111(2) & \mathrm{O}(3) & \mathrm{C}(5) & \mathrm{H}(9) & 104(3) \\ \mathrm{O}(3) & \mathrm{C}(5) & \mathrm{H}(10) & 109(2) & \mathrm{H}(8) & \mathrm{C}(5) & \mathrm{H}(9) & 110(4) \\ \mathrm{H}(8) & \mathrm{C}(5) & \mathrm{H}(10) & 108(4) & \mathrm{H}(9) & \mathrm{C}(5) & \mathrm{H}(10) & 112(4) \\ \mathrm{C}(2) & \mathrm{C}(6) & \mathrm{H}(11) & 114(1) & \mathrm{C}(2) & \mathrm{C}(6) & \mathrm{H}(12) & 111(1) \\ \mathrm{C}(7) & \mathrm{C}(6) & \mathrm{H}(11) & 107(2) & \mathrm{C}(7) & \mathrm{C}(6) & \mathrm{H}(12) & 110(1) \\ \mathrm{H}(11) & \mathrm{C}(6) & \mathrm{H}(12) & 103(2) & \mathrm{C}(6) & \mathrm{C}(7) & \mathrm{H}(13) & 109(2) \\ \mathrm{C}(6) & \mathrm{C}(7) & \mathrm{H}(14) & 114(2) & \mathrm{C}(8) & \mathrm{C}(7) & \mathrm{H}(13) & 109(2) \\ \mathrm{C}(8) & \mathrm{C}(7) & \mathrm{H}(14) & 105(2) & \mathrm{H}(13) & \mathrm{C}(7) & \mathrm{H}(14) & 104(4) \\ \mathrm{C}(8) & \mathrm{C}(9) & \mathrm{H}(15) & 120(2) & \mathrm{C}(10) & \mathrm{C}(9) & \mathrm{H}(15) & 117(2) \\ \mathrm{C}(9) & \mathrm{C}(10) & \mathrm{H}(16) & 115(1) & \mathrm{C}(11) & \mathrm{C}(10) & \mathrm{H}(16) & 124(1) \\ \mathrm{C}(10) & \mathrm{C}(11) & \mathrm{H}(17) & 123(1) & \mathrm{C}(12) & \mathrm{C}(11) & \mathrm{H}(17) & 117(2) \\ \mathrm{C}(11) & \mathrm{C}(12) & \mathrm{H}(18) & 116(1) & \mathrm{C}(13) & \mathrm{C}(12) & \mathrm{H}(18) & 122(1)\end{array}$

Table 7. Torsion Angles( $\left.{ }^{0}\right)$

\begin{tabular}{|c|c|c|c|c|c|c|c|c|c|}
\hline tom & atom & atom & atom & angle & atom & atom & atom & atom & angle \\
\hline (1) & ) & 2 ) & $C(3)$ & (2) & & & $C$ & 7 & (2) \\
\hline$O(1)$ & $C(1)$ & $C(13)$ & $C(8)$ & $98.3(3)$ & $O(1)$ & $C(1)$ & $C(13)$ & $C(12)$ & $-82.0(3)$ \\
\hline$O(2)$ & $C(3)$ & $O(3)$ & $C(5)$ & $-120.6(2)$ & $O(2)$ & $C(3)$ & $C(2)$ & $C(1)$ & $60.9(2)$ \\
\hline$O(2)$ & $C(3)$ & $C(2)$ & $C(6)$ & $-176.5(2)$ & $O(3)$ & $C(3)$ & $O(2)$ & $C(4)$ & $67.6(3)$ \\
\hline$O(3)$ & $\mathrm{C}($ & $C(2)$ & $C(1)$ & 179. & $O(3)$ & $\mathrm{C}($ & $C(2)$ & ) & $-57.5(3)$ \\
\hline$C(1)$ & $C(2)$ & $C(6)$ & $C(7)$ & -66. & $C(1)$ & $C(13)$ & $C(8)$ & $C(7)$ & $1.8(4)$ \\
\hline$C(1)$ & $C(13)$ & $C(8)$ & $C(9)$ & 179.2 & $C(1)$ & $C(13)$ & $C(12)$ & $C(11)$ & -179 \\
\hline$C(2)$ & $C(1)$ & $C(13)$ & $C(8)$ & -20 & $C(2)$ & $C(1)$ & $C(13)$ & $C(12)$ & 158 \\
\hline$C(2)$ & $C(3)$ & $O(2)$ & $C(4)$ & $-172.8(2)$ & $C(2)$ & $C(3)$ & $O(3)$ & $C(5)$ & $121.4(2)$ \\
\hline$C(2)$ & $C(6)$ & $C(7)$ & $C(8)$ & $46.8(3)$ & $C(3)$ & $C(2)$ & $C(1)$ & $C(13)$ & $177.5(2)$ \\
\hline$C(3)$ & $C(2)$ & $C(6)$ & $C(7)$ & 169.71 & $C(6)$ & $C(2)$ & $C(1)$ & $C(13)$ & $52.2(3)$ \\
\hline$C(6)$ & $C(7)$ & $C(8)$ & $C(9)$ & 167. & $C(6)$ & $C(7)$ & $C(8)$ & $C(13)$ & $-15.0(4)$ \\
\hline$C(7)$ & $C(8)$ & $C(9)$ & $C(10)$ & $177.4(3)$ & $C(7)$ & $C(8)$ & $C(13)$ & $C(12)$ & $-178.0(3)$ \\
\hline$C(8)$ & $C(9)$ & $C(10)$ & $C(11)$ & $0.9(5)$ & $C(8)$ & $C(13)$ & $C(12)$ & $C(11)$ & $0.3(4)$ \\
\hline$C(9)$ & $C(8)$ & $C(13)$ & $C(12)$ & -0 & $C(9)$ & & & $C(12)$ & $-1.2(5)$ \\
\hline$C(10)$ & $C(9)$ & $C(8)$ & $C(13)$ & $-0.1(4)$ & $C(10)$ & $C(11)$ & $C(12)$ & $C(13)$ & $0.6(5)$ \\
\hline
\end{tabular}

Table 8. Non-bonded Contacts out to $3.60 \AA$ 


$\begin{array}{llllll}\text { atom } & \text { atom } & \text { distance } & \text { atom } & \text { atom } & \text { distance } \\ \mathrm{O}(1) & \mathrm{O}(2)^{1)} & 2.805(2) & \mathrm{O}(1) & \mathrm{C}(5)^{2)} & 3.378(4) \\ \mathrm{O}(3) & \mathrm{C}(5)^{3)} & 3.569(4) & & & \end{array}$

\section{Symmetry operations}
(1) $-\mathrm{X}, \mathrm{Y}+1 / 2,-\mathrm{Z}+1$
(3) $-\mathrm{X}+1, \mathrm{Y}-1 / 2,-\mathrm{Z}+1$

$-\mathrm{X}+1, \mathrm{Y}+1 / 2,-\mathrm{Z}+1$ 